\title{
Prenex normal form theorems in semi-classical arithmetic
}

\author{
Makoto Fujiwara ${ }^{* \dagger}$ and Taishi Kurahashi ${ }^{\ddagger \S}$
}

September 9, 2020

\begin{abstract}
Akama et al. [1] systematically studied an arithmetical hierarchy of the law of excluded middle and related principles in the context of first-order arithmetic. In that paper, they first provide a prenex normal form theorem as a justification of their semi-classical principles restricted to prenex formulas. However, there are some errors in their proof. In this paper, we provide a simple counterexample of their prenex normal form theorem [1, Theorem 2.7], then modify it in an appropriate way. In addition, we characterize several prenex normal form theorems with respect to semi-classical arithmetic.
\end{abstract}

\section{Introduction}

Prenex normal form theorem is one of the most basic theorems on theories based on classical first-order predicate logic. In contrast, it does not hold for intuitionistic theories in general. Therefore it does not make sense to consider an arithmetical hierarchy in an intuitionistic theory. On the other hand, if one reasons in some semi-classical arithmetic which lies in-between classical arithmetic and intuitionistic arithmetic, one can take an equivalent formula of the prenex normal form for any formula with low complexity. Akama et al. 1] introduces the classes of formulas $\mathrm{E}_{k}$ and $\mathrm{U}_{k}$ which corresponds to the classes of classical $\Sigma_{k}$ and $\Pi_{k}$ formulas respectively, and showed that the former is equivalent to the class of formulas of $\Sigma_{k}$ form and the latter is so for $\Pi_{k}$ over some semi-classical arithmetic respectively. This prenex normal form theorem justifies their investigation on the arithmetical

\footnotetext{
*Email: makotofujiwara@meiji.ac.jp

${ }^{\dagger}$ School of Science and Technology, Meiji University, 1-1-1 Higashi-Mita, Tama-ku, Kawasaki-shi, Kanagawa 214-8571, Japan.

${ }^{\ddagger}$ Email: kurahashi@people.kobe-u.ac.jp

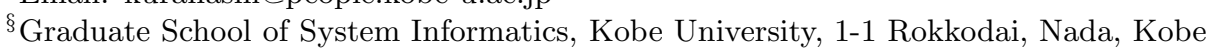
657-8501, Japan.
} 
hierarchy in the context of intuitionistic first-order arithmetic. Unfortunately, however, there are some crucial errors in their proof of the prenex normal form theorem [1, Theorem 2.7]. In this paper, we revisit their formulation and modify their prenex normal form theorem in an appropriate way.

In Section 2, we recall the definitions and basic properties. In Section 3. we provide a simple counterexample of [1, Theorem 2.7]. In Section 5. we show the corrected version of the prenex normal form theorem (see Theorem [5.3). In addition, we also present the simplified version of the prenex normal form theorem for formulas which do not contain the disjunction (see Theorem 5.7). In Section 6, we carry out some generalization of a well-known result that classical arithmetic is $\Pi_{2}$-conservative over intuitionistic arithmetic with respect to semi-classical arithmetic. In Section 7 , using the generalized conservation result in Section 6, we characterize several prenex normal form theorems with respect to semi-classical arithmetic. In particular, among other things, we show that for any theory $T$ in-between intuitionistic arithmetic and classical arithmetic, $T$ proves a semi-classical principle $\left(\Pi_{k} \vee \Pi_{k}\right)$-DNE if and only if $T$ satisfies the prenex normal form theorem for $\mathrm{U}_{k^{\prime}}$ and $\Pi_{k^{\prime}}$ for all $k^{\prime} \leq k$ (see Theorem 7.3 ).

Throughout this paper, we work basically over intuitionistic arithmetic. When we use some principle (including induction hypothesis [I.H.]) which is not available in intuitionistic arithmetic, it will be exhibited explicitly. As regards basic reasoning over intuitionistic first-order logic, we refer the reader to see [9, Section 6.2].

\section{Preparation}

Throughout this paper, we work with a standard formulation of intuitionistic arithmetic HA described e.g. in [7, Section 1.3], which has function symbols for all primitive recursive functions. We work in the language containing all the logical constants $\forall, \exists, \rightarrow, \wedge, \vee, \perp$. Let $T$ denote a theory (e.g. HA), and $\mathrm{P}$ and $\mathrm{Q}$ denote schemata (e.g. logical principles). Then $T+\mathrm{P}$ denotes the theory obtained from $T$ by adding $\mathrm{P}$ into the axioms. In particular, the classical variant PA is defined as HA + LEM, where LEM is the axiom scheme of the law of excluded middle. We write $T \vdash \mathrm{Q}$ (or $T$ proves $\mathrm{Q}$ ) if any instance of $\mathrm{Q}$ is provable in $T$. We write $T \vdash \mathrm{P}+\mathrm{Q}$ if $T \vdash \mathrm{P}$ and $T \vdash \mathrm{Q}$.

Notation 1. For a formula $\varphi, \mathrm{FV}(\varphi)$ denotes the set of free variables in $\varphi$. Quantifier-free formulas are denoted with subscript "qf" as $\varphi_{\mathrm{qf}}$. In addition, a list of variables is denoted with an over-line as $\bar{x}$. In particular, a list of quantifiers of the same kind is denoted as $\exists \bar{x}$ and $\forall \bar{x}$ respectively.

Definition 2.1. The classes $\Sigma_{k}$ and $\Pi_{k}$ of formulas are defined as follows:

- $\Sigma_{0}$, as well as $\Pi_{0}$, is the class of all quantifier-free formulas; 
- $\Pi_{k+1}$ is the class of all formulas of form $Q_{1} \overline{x_{1}} \cdots Q_{k+1} \overline{x_{k+1}} \varphi_{\mathrm{qf}}$;

- $\Sigma_{k+1}$ is the class of all formulas of form $Q_{1}^{\prime} \overline{x_{1}} \cdots Q_{k+1}^{\prime} \overline{x_{k+1}} \varphi_{\mathrm{qf}}$;

where $Q_{i}$ represents $\forall$ for odd $i$ and $\exists$ for even $i$ and $Q_{i}^{\prime}$ represents $\exists$ for odd $i$ and $\forall$ for even $i$. Following [1], we define the classes $\Sigma_{k}$ and $\Pi_{k}$ in the non-cumulative manner (namely, each $Q_{i} \overline{x_{i}}$ and $Q_{i}^{\prime} \overline{x_{i}}$ must not be empty). A formula $\varphi$ is of prenex normal form if $\varphi \in \Sigma_{k} \cup \Pi_{k}$ for some $k$.

Remark 2.2. Since the list of variables can be contracted into one variable in HA by using a fixed primitive recursive pairing function (see e.g. [7, 1.3.9]), one may assume that for each natural number $k>0$, a formula in $\Sigma_{k}$ is of form $\exists x \varphi(x)$ with some $\varphi(x) \in \Pi_{k-1}$ and a formula in $\Pi_{k}$ is of form $\forall x \psi(x)$ with some $\psi(x) \in \Sigma_{k-1}$ without loss of generality.

Lemma 2.3. Let $k$ be a natural number. Let $\varphi$ be in $\Pi_{k}$ and $\psi$ be in $\Sigma_{k}$. Then, for all natural numbers $i, j$, there exist $\varphi^{\prime}, \psi^{\prime} \in \Pi_{k+i}$ and $\varphi^{\prime \prime}, \psi^{\prime \prime} \in$ $\Sigma_{k+j}$ such that $\mathrm{FV}(\varphi)=\mathrm{FV}\left(\varphi^{\prime}\right)=\mathrm{FV}\left(\varphi^{\prime \prime}\right), \mathrm{FV}(\psi)=\mathrm{FV}\left(\psi^{\prime}\right)=\mathrm{FV}\left(\psi^{\prime \prime}\right)$, $\mathrm{HA} \vdash \varphi \leftrightarrow \varphi^{\prime} \leftrightarrow \varphi^{\prime \prime}$ and $\mathrm{HA} \vdash \psi \leftrightarrow \psi^{\prime} \leftrightarrow \psi^{\prime \prime}$.

Proof. Straightforward by the fact that

$$
\mathrm{HA} \vdash \xi \leftrightarrow \forall z \xi \leftrightarrow \exists z \xi
$$

for any $z \notin \mathrm{FV}(\xi)$.

Definition 2.4. For a class $\Gamma$ of formulas, $\Gamma(\bar{x})$ denotes the class of formulas $\varphi$ in $\Gamma$ such that $\mathrm{FV}(\varphi) \subseteq\{\bar{x}\}$.

Remark 2.5. In the light of Lemma 2.3, throughout this paper, we identify the classes $\Sigma_{k}$ and $\Pi_{k}$ with the classes defined as in Definition 2.1 with allowing the quantifiers $Q_{i}$ and $Q_{i}^{\prime}$ to be empty. Under this identification, for all $k$ and $k^{\prime}$ such that $k<k^{\prime}, \Pi_{k}(\bar{x})$ and $\Sigma_{k}(\bar{x})$ are considered to be sub-classes of $\Sigma_{k^{\prime}}(\bar{x}) \cap \Pi_{k^{\prime}}(\bar{x})$. We frequently use this property in what follows.

Recall the logical principles from [1] and related principles:

Definition 2.6. Let $\Gamma$ and $\Gamma^{\prime}$ be classes of formulas.

- $\Gamma$-LEM : $\forall x(\varphi(x) \vee \neg \varphi(x))$ where $\varphi(x) \in \Gamma(x)$.

- $\Gamma$-DML : $\forall x(\neg(\varphi(x) \wedge \psi(x)) \rightarrow \neg \varphi(x) \vee \neg \psi(x))$ where $\varphi(x), \psi(x) \in$ $\Gamma(x)$.

- $\Gamma$-DNE : $\forall x(\neg \neg \varphi(x) \rightarrow \varphi(x))$ where $\varphi(x) \in \Gamma(x)$.

- $\left(\Gamma \vee \Gamma^{\prime}\right)$-DNE : $\forall x(\neg \neg(\varphi(x) \vee \psi(x)) \rightarrow \varphi(x) \vee \psi(x))$ where $\varphi(x) \in$ $\Gamma(x)$ and $\psi(x) \in \Gamma^{\prime}(x)$. 
- $\Gamma$-DNS : $\forall x(\forall y \neg \neg \varphi(x, y) \rightarrow \neg \neg \forall y \varphi(x, y))$ where $\varphi(x, y) \in \Gamma(x, y)$.

- Let $\mathrm{P} \in\left\{\Gamma\right.$-LEM, $\Gamma$-DML, $\Gamma$-DNE, $\left(\Gamma \vee \Gamma^{\prime}\right)$-DNE, $\Gamma$-DNS $\}$.

$\neg \neg \mathrm{P}: \neg \neg \xi$ where $\xi$ is an instance of $\mathrm{P}$.

Note that our logical principles are equivalent also to those defined with lists of quantifiers of the same kind (cf. Remark 2.2).

Remark 2.7. One has to care about the formulation of the double negated variants. That is, one has to take the double negations of the universal closure of the original logical principles as in Definition 2.6. The double negated variants defined as such are not provable in HA, which has been overlooked in the proof of [1, Theorem 2.7] (see also Section [3). In fact, one may think of the double negated versions as variants of the double negation shift principle (see [3]). In addition, our double negated versions are equivalent to (the universal closures of) those with allowing free variables (cf. [3, Remark 2.5]).

Remark 2.8. For any class $\Gamma$ of formulas, $\Gamma$-DNS is intuitionistically equivalent to $\neg \neg \Gamma$-DNS since

$$
\begin{aligned}
& \forall x(\forall y \neg \neg \varphi \rightarrow \neg \neg \forall y \varphi) \\
& \longleftrightarrow \quad \forall x \neg \neg(\forall y \neg \neg \varphi \rightarrow \neg \neg \forall y \varphi) \\
& \longleftrightarrow \quad \neg \neg \forall x \neg \neg(\forall y \neg \neg \varphi \rightarrow \neg \neg \forall y \varphi) \\
& \longleftrightarrow \quad \neg \neg \forall x(\forall y \neg \neg \varphi \rightarrow \neg \neg \forall y \varphi) .
\end{aligned}
$$

Next we reformulate the classes of formulas studied in [1]. The classes $\mathrm{F}_{k}, \mathrm{U}_{k}$ and $\mathrm{E}_{k}$ in Definition 2.11 below were dealt with in [1] informally. Here we shall introduce them and two additional classes $\mathrm{U}_{k}^{+}$and $\mathrm{E}_{k}^{+}$in a formal manner.

Definition 2.9. An alternation path is a finite sequence of + and - in which + and - appear alternatively. For an alternation path $s$, let $i(s)$ denote the first symbol of $s$ if $s \not \equiv\langle\rangle$ (empty sequence); $\times$ if $s \equiv\langle\rangle$. Let $s^{\perp}$ denote an alternation path which is obtained by switching + and - in $s$, and let $l(s)$ denote the length of $s$.

Definition 2.10. For a formula $\varphi$, the set of alternation paths $\operatorname{Alt}(\varphi)$ of $\varphi$ is defined as follows:

- If $\varphi$ is quantifier-free, then $\operatorname{Alt}(\varphi):=\{\langle\rangle\}$;

- Otherwise, $\operatorname{Alt}(\varphi)$ is defined inductively by the following rule:

- If $\varphi \equiv \neg \varphi_{1}$, then $\operatorname{Alt}(\varphi):=\left\{s^{\perp} \mid s \in \operatorname{Alt}\left(\varphi_{1}\right)\right\}$

- If $\varphi \equiv \varphi_{1} \wedge \varphi_{2}$ or $\varphi \equiv \varphi_{1} \vee \varphi_{2}$, then $\operatorname{Alt}(\varphi):=\operatorname{Alt}\left(\varphi_{1}\right) \cup \operatorname{Alt}\left(\varphi_{2}\right)$;

- If $\varphi \equiv \varphi_{1} \rightarrow \varphi_{2}$, then $\operatorname{Alt}(\varphi):=\left\{s^{\perp} \mid s \in \operatorname{Alt}\left(\varphi_{1}\right)\right\} \cup \operatorname{Alt}\left(\varphi_{2}\right)$; 
- If $\varphi \equiv \forall x \varphi_{1}$, then $\operatorname{Alt}(\varphi):=\left\{s \mid s \in \operatorname{Alt}\left(\varphi_{1}\right)\right.$ and $\left.i(s) \equiv-\right\} \cup$ $\left\{-s \mid s \in \operatorname{Alt}\left(\varphi_{1}\right)\right.$ and $\left.i(s) \not \equiv-\right\}$;

- If $\varphi \equiv \exists x \varphi_{1}$, then $\operatorname{Alt}(\varphi):=\left\{s \mid s \in \operatorname{Alt}\left(\varphi_{1}\right)\right.$ and $\left.i(s) \equiv+\right\} \cup$ $\left\{+s \mid s \in \operatorname{Alt}\left(\varphi_{1}\right)\right.$ and $\left.i(s) \not \equiv+\right\}$.

In addition, for a formula $\varphi$, the degree $\operatorname{deg}(\varphi)$ of $\varphi$ is defined as

$$
\operatorname{deg}(\varphi):=\max \{l(s) \mid s \in \operatorname{Alt}(\varphi)\} .
$$

Definition 2.11. The classes $\mathrm{F}_{k}, \mathrm{U}_{k}, \mathrm{E}_{k}$ (from [1, Definition 2.4]), $\mathrm{U}_{k}^{+}$and $\mathrm{E}_{k}^{+}$of formulas are defined as follows:

- $\mathrm{F}_{k}:=\{\varphi \mid \operatorname{deg}(\varphi)=k\} ;$

- $\mathrm{U}_{0}:=\mathrm{E}_{0}:=\mathrm{F}_{0}$

- $\mathrm{U}_{k+1}:=\left\{\varphi \in \mathrm{F}_{k+1} \mid i(s) \equiv-\right.$ for all $s \in \operatorname{Alt}(\varphi)$ such that $l(s)=$ $k+1\}$

- $\mathrm{E}_{k+1}:=\left\{\varphi \in \mathrm{F}_{k+1} \mid i(s) \equiv+\right.$ for all $s \in \operatorname{Alt}(\varphi)$ such that $l(s)=$ $k+1\}$

- $\mathrm{U}_{k}^{+}:=\mathrm{U}_{k} \cup \bigcup_{i<k} \mathrm{~F}_{i}$;

- $\mathrm{E}_{k}^{+}:=\mathrm{E}_{k} \cup \bigcup_{i<k} \mathrm{~F}_{i}$.

Remark 2.12. A similar property as Lemma 2.3 also holds for $\mathrm{U}_{k}^{+}$and $\mathrm{E}_{k}^{+}$: for any $\varphi \in \mathrm{U}_{k}^{+}$and $\psi \in \mathrm{E}_{k}^{+}$, there exist $\varphi^{\prime} \in \mathrm{U}_{k}$ and $\psi^{\prime} \in \mathrm{E}_{k}$ such that $\mathrm{FV}(\varphi)=\mathrm{FV}\left(\varphi^{\prime}\right), \mathrm{FV}(\psi)=\mathrm{FV}\left(\psi^{\prime}\right), \mathrm{HA} \vdash \varphi \leftrightarrow \varphi^{\prime}$ and $\mathrm{HA} \vdash \psi \leftrightarrow \psi^{\prime}$.

Note $\mathrm{F}_{0}=\Sigma_{0}=\Pi_{0}$. For each formula $\varphi \in \mathrm{E}_{k}$ (resp. $\psi \in \mathrm{U}_{k}$ ) of PA, one can take a formula $\varphi^{\prime} \in \Sigma_{k}$ (resp. $\psi^{\prime} \in \Pi_{k}$ ) of PA which is equivalent to $\varphi$ (resp. $\psi$ ) over PA. On the other hand, this is not the case for HA. In what follows, we study what kind of semi-classical arithmetic in-between PA and HA captures this property for each $k$. In fact, Akama et al. [1] has already undertaken this. In particular, [1, Theorem 2.7] asserts the following:

1. For any $\varphi \in \mathrm{E}_{k}$, there exists $\varphi^{\prime} \in \Sigma_{k}$ such that

$$
\mathrm{HA}+\Sigma_{k}-\mathrm{DNE} \vdash \varphi \leftrightarrow \varphi^{\prime} .
$$

2. For any $\varphi \in \mathrm{U}_{k}$, there exists $\varphi^{\prime} \in \Pi_{k}$ such that

$$
\mathrm{HA}+\left(\Pi_{k} \vee \Pi_{k}\right) \text {-DNE } \vdash \varphi \leftrightarrow \varphi^{\prime} .
$$

However, the first assertion is wrong as we show in Section 3 . 


\section{A counter example}

Recall that [1, Theorem 2.7] asserts that for any $\varphi \in \mathrm{E}_{k}$, there exists $\varphi^{\prime} \in \Sigma_{k}$ such that

$$
\mathrm{HA}+\Sigma_{k} \text {-DNE } \vdash \varphi \leftrightarrow \varphi^{\prime} .
$$

However, there are some errors in the proof. In particular, in [1, page 5, lines 15-17], it is written that "Since the double negations of DNE is intuitionistically provable, $\vdash_{\mathrm{HA}} \neg \neg A_{0} \leftrightarrow \neg \neg \exists x_{0} . C_{0}$ (which means $\mathrm{HA} \vdash$ $\neg \neg A_{0} \leftrightarrow \neg \neg \exists x_{0} C_{0}$ in our notation) ". As studied in [3], however, the double negations of (the universal closure of) DNE is not provable in HA, and hence, their proof actually uses some double negated logical principles in the sense of Definition 2.6. Our counterexample below shows that such a use of some additional principle is unavoidable.

Recall the arithmetical form of Church's thesis from [7, 3.2.14]:

$$
\mathrm{CT}_{0}: \forall x \exists y \varphi(x, y) \rightarrow \exists e \forall x \exists v(\mathrm{~T}(e, x, v) \wedge \varphi(x, \mathrm{U}(v))),
$$

where $\mathrm{T}$ and $\mathrm{U}$ are the standard primitive recursive predicate and function from the Kleene normal form theorem. Note that $\mathrm{CT}_{0}$ is a sort of combination of so-called Church's thesis stating that every function is recursive and the countable choice principle (see [8, 4.3.2]).

Proposition 3.1. The following sentence

$$
\varphi_{0}: \equiv \neg \forall x(\neg \exists u(\mathrm{~T}(x, x, u) \wedge \mathrm{U}(u)=0) \vee \neg \exists u(\mathrm{~T}(x, x, u) \wedge \mathrm{U}(u) \neq 0))
$$

is not equivalent to any sentence $\varphi_{0}^{\prime} \in \Sigma_{1}$ over $\mathrm{HA}+\Sigma_{1}$-DNE.

Proof. We first claim that $\mathrm{HA}+\mathrm{CT}_{0}$ proves $\varphi_{0}$. For the sake of contradiction, assume

$$
\forall x(\neg \exists u(\mathrm{~T}(x, x, u) \wedge \mathrm{U}(u)=0) \vee \neg \exists u(\mathrm{~T}(x, x, u) \wedge \mathrm{U}(u) \neq 0))
$$

and reason in $\mathrm{HA}+\mathrm{CT}_{0}$. Since $\varphi_{1} \vee \varphi_{2} \leftrightarrow \exists k\left(\left(k=0 \rightarrow \varphi_{1}\right) \wedge\left(k \neq 0 \rightarrow \varphi_{2}\right)\right)$ (see [7, 1.3.7]), by $\mathrm{CT}_{0}$, there exists $e$ such that

$$
\forall x \exists v\left(\begin{array}{l}
\mathrm{T}(e, x, v) \\
\wedge(\mathrm{U}(v)=0 \rightarrow \neg \exists u(\mathrm{~T}(x, x, u) \wedge \mathrm{U}(u)=0)) \\
\wedge(\mathrm{U}(v) \neq 0 \rightarrow \neg \exists u(\mathrm{~T}(x, x, u) \wedge \mathrm{U}(u) \neq 0))
\end{array}\right) .
$$

In particular, for that $e$, there exists $v_{e}$ such that $\mathrm{T}\left(e, e, v_{e}\right)$,

$$
\mathrm{U}\left(v_{e}\right)=0 \rightarrow \neg \exists u(\mathrm{~T}(e, e, u) \wedge \mathrm{U}(u)=0)
$$

and

$$
\mathrm{U}\left(v_{e}\right) \neq 0 \rightarrow \neg \exists u(\mathrm{~T}(e, e, u) \wedge \mathrm{U}(u) \neq 0) .
$$


Since $\mathrm{U}\left(v_{e}\right)=0 \vee \mathrm{U}\left(v_{e}\right) \neq 0$, we obtain a contradiction straightforwardly.

If $\varphi_{0}$ is equivalent to some sentence $\varphi_{0}^{\prime} \in \Sigma_{1}$ over $\mathrm{HA}+\Sigma_{1}$-DNE, we have $\mathrm{HA}+\Sigma_{1}$-DNE $+\mathrm{CT}_{0} \vdash \varphi_{0}^{\prime}$ from the above claim. Since $\varphi_{0}^{\prime} \in \Sigma_{1}$, by the soundness of Kleene realizability (see [7, 3.2.22]), we have that

$$
\mathrm{HA}+\Sigma_{1}-\mathrm{DNE} \vdash \varphi_{0}^{\prime},
$$

and hence, $\mathrm{HA}+\Sigma_{1}$-DNE $\vdash \varphi_{0}$. On the other hand, since

$$
\forall x(\neg(\exists u(\mathrm{~T}(x, x, u) \wedge \mathrm{U}(u)=0) \wedge \exists u(\mathrm{~T}(x, x, u) \wedge \mathrm{U}(u) \neq 0)))
$$

is provable in $\mathrm{HA}$, we have $\mathrm{HA}+\Sigma_{1}$-DML $\vdash$ (1). Therefore we have

$$
\mathrm{HA}+\Sigma_{1}-\mathrm{DNE}+\Sigma_{1}-\mathrm{DML} \vdash \perp,
$$

and hence, $\mathrm{PA} \vdash \perp$, which is a contradiction.

Remark 3.2. One can easily see that $\varphi_{0}$ in Proposition 3.1 is in $\mathrm{E}_{1}$. Thus Proposition 3.1] shows that $\varphi_{0}$ is a counterexample of [1, Theorem 2.7] for $k=1$.

\section{Basic lemmata}

In this section, we show several lemmata which we use in the proofs of our prenex normal form theorems.

Lemma 4.1. For any logical principle $\mathrm{P}$ in Definition 2.6 and any formula $\varphi$ (possibly containing free variables), if $\mathrm{HA}+\mathrm{P} \vdash \varphi$, then $\mathrm{HA}+\neg \neg \mathrm{P} \vdash \neg \neg \varphi$.

Proof. Assume $\mathrm{HA}+\mathrm{P} \vdash \varphi$. Then there exists finite instances $\psi_{1}, \ldots, \psi_{k}$ of $\mathrm{P}$ such that $\mathrm{HA}+\psi_{1}+\cdots+\psi_{k} \vdash \varphi$. Since $\mathrm{HA}$ satisfies the deduction theorem, we have that HA proves $\psi_{1} \wedge \cdots \wedge \psi_{k} \rightarrow \varphi$, and hence, $\neg \neg\left(\psi_{1} \wedge \cdots \wedge \psi_{k} \rightarrow \varphi\right)$, which is equivalent to $\neg \neg \psi_{1} \wedge \cdots \wedge \neg \neg \psi_{k} \rightarrow \neg \neg \varphi$. Then we have $\mathrm{HA}+\neg \neg \mathrm{P} \vdash$ $\neg \neg \varphi$.

Corollary 4.2. For any logical principle $\mathrm{P}$ in Definition 2.6 and any formulas $\varphi_{1}$ and $\varphi_{2}$ (possibly containing free variables), if $\mathrm{HA}+\mathrm{P} \vdash \varphi_{1} \leftrightarrow \varphi_{2}$, then $\mathrm{HA}+\neg \neg \mathrm{P} \vdash \neg \neg \varphi_{1} \leftrightarrow \neg \neg \varphi_{2}$.

Proof. Immediate from Lemma 4.1 and the fact that $\neg \neg\left(\varphi_{1} \leftrightarrow \varphi_{2}\right)$ is intuitionistically equivalent to $\neg \neg \varphi_{1} \leftrightarrow \neg \neg \varphi_{2}$.

Lemma 4.3. Let $k$ be a natural number. Let $\varphi_{1}$ and $\varphi_{2}$ be formulas in $\Sigma_{k}$, and let $\varphi_{3}$ and $\varphi_{4}$ be formulas in $\Pi_{k}$. Then the following hold:

1. There exists a formula $\varphi \in \Sigma_{k}$ such that $\mathrm{FV}(\varphi)=\mathrm{FV}\left(\varphi_{1}\right) \cup \operatorname{FV}\left(\varphi_{2}\right)$ and $\mathrm{HA} \vdash \varphi \leftrightarrow \varphi_{1} \wedge \varphi_{2}$; 
2. There exists a formula $\varphi^{\prime} \in \Pi_{k}$ such that $\mathrm{FV}\left(\varphi^{\prime}\right)=\mathrm{FV}\left(\varphi_{3}\right) \cup \mathrm{FV}\left(\varphi_{4}\right)$ and $\mathrm{HA} \vdash \varphi^{\prime} \leftrightarrow \varphi_{3} \wedge \varphi_{4}$.

Proof. Straightforward by simultaneous induction on $k$.

Lemma 4.4. For any formulas $\varphi_{1}$ and $\varphi_{2}$ in $\Sigma_{k}$, there exists a formula $\varphi \in \Sigma_{k}$ such that $\mathrm{FV}(\varphi)=\mathrm{FV}\left(\varphi_{1}\right) \cup \mathrm{FV}\left(\varphi_{2}\right)$ and $\mathrm{HA} \vdash \varphi \leftrightarrow \varphi_{1} \vee \varphi_{2}$.

Proof. Note that $\varphi_{1} \vee \varphi_{2}$ is equivalent to

$$
\exists k\left(\left(k=0 \rightarrow \varphi_{1}\right) \wedge\left(k \neq 0 \rightarrow \varphi_{2}\right)\right)
$$

over HA (see [7, 1.3.7]). Since $\varphi_{\mathrm{qf}} \rightarrow \exists x \psi(x)$ and $\varphi_{\mathrm{qf}} \rightarrow \forall x \psi(x)$ are equivalent to $\exists x\left(\varphi_{\mathrm{qf}} \rightarrow \psi(x)\right)$ and $\forall x\left(\varphi_{\mathrm{qf}} \rightarrow \psi(x)\right)$ respectively over HA when $x \notin \mathrm{FV}\left(\varphi_{\mathrm{qf}}\right)$, our assertion follows from Lemma 4.3 straightforwardly.

Lemma 4.5. Let $k$ be a natural number greater than 0 .

1. If $\varphi_{1} \wedge \varphi_{2}$ is in $\mathrm{U}_{k}^{+}\left(\right.$resp. $\left.\mathrm{E}_{k}^{+}\right)$if and only if both of $\varphi_{1}$ and $\varphi_{2}$ are in $\mathrm{U}_{k}^{+}\left(\right.$resp. $\left.\mathrm{E}_{k}^{+}\right)$.

2. $\varphi_{1} \vee \varphi_{2}$ is in $\mathrm{U}_{k}^{+}$(resp. $\mathrm{E}_{k}^{+}$) if and only if both of $\varphi_{1}$ and $\varphi_{2}$ are in $\mathrm{U}_{k}^{+}\left(\right.$resp. $\left.\mathrm{E}_{k}^{+}\right)$.

3. $\varphi_{1} \rightarrow \varphi_{2}$ is in $\mathrm{U}_{k}^{+}$(resp. $\left.\mathrm{E}_{k}^{+}\right)$if and only if $\varphi_{1}$ is in $\mathrm{E}_{k}^{+}$(resp. $\mathrm{U}_{k}^{+}$) and $\varphi_{2}$ is in $\mathrm{U}_{k}^{+}$(resp. $\mathrm{E}_{k}^{+}$).

4. $\forall x \varphi_{1}$ is in $\mathrm{U}_{k}^{+}$if and only if $\varphi_{1}$ is in $\mathrm{U}_{k}^{+}$.

5. $\exists x \varphi_{1}$ is in $\mathrm{E}_{k}^{+}$if and only if $\varphi_{1}$ is in $\mathrm{E}_{k}^{+}$.

6. $\forall x \varphi_{1}$ is in $\mathrm{E}_{k+1}^{+}$if and only if it is in $\mathrm{U}_{k}^{+}$.

7. $\exists x \varphi_{1}$ is in $\mathrm{U}_{k+1}^{+}$if and only if it is in $\mathrm{E}_{k}^{+}$.

Proof. (11): Assume $\varphi_{1} \wedge \varphi_{2} \in \mathrm{U}_{k}^{+}$. Then $l(s) \leq k$ for all $s \in \operatorname{Alt}\left(\varphi_{1} \wedge \varphi_{2}\right)=$ $\operatorname{Alt}\left(\varphi_{1}\right) \cup \operatorname{Alt}\left(\varphi_{2}\right)$.

- If $l(s)<k$ for all $s \in A l t\left(\varphi_{1}\right)$, then $\varphi_{1}$ is in $\bigcup_{i<k} \mathrm{~F}_{i} \subseteq \mathrm{U}_{k}^{+}$.

- Otherwise, there is $s_{0} \in \operatorname{Alt}\left(\varphi_{1}\right)$ such that $l\left(s_{0}\right)=k$. Then, since $\varphi_{1} \wedge \varphi_{2} \notin \bigcup_{i<k} \mathrm{~F}_{i}$, we have $\varphi_{1} \wedge \varphi_{2} \in \mathrm{U}_{k}$. Then, for each $s \in \operatorname{Alt}\left(\varphi_{1}\right)$ such that $l(s)=k$, we have $i(s) \equiv-$ since $s \in \operatorname{Alt}\left(\varphi_{1} \wedge \varphi_{2}\right)$. Thus $\varphi_{1} \in \mathrm{U}_{k} \subseteq \mathrm{U}_{k}^{+}$. 
We also have $\varphi_{2} \in \mathrm{U}_{k}^{+}$in the same manner.

For the converse direction, assume that $\varphi_{1}$ and $\varphi_{2}$ are in $\mathrm{U}_{k}^{+}$. Then, for all $s \in \operatorname{Alt}\left(\varphi_{1} \wedge \varphi_{2}\right)$, since $s \in \operatorname{Alt}\left(\varphi_{1}\right)$ or $s \in \operatorname{Alt}\left(\varphi_{2}\right)$, we have $l(s) \leq k$, in particular, $i(s) \equiv-$ if $l(s)=k$. Thus $\varphi_{1} \wedge \varphi_{2}$ is in $\mathrm{U}_{k}^{+}$.

As for the case of $\mathrm{E}_{k}^{+}$, an analogous proof works.

(2): Analogous to (11).

(3)): Assume $\varphi_{1} \rightarrow \varphi_{2} \in \mathrm{U}_{k}^{+}$. Let $s$ be in $\operatorname{Alt}\left(\varphi_{1}\right)$. By the definition of $\operatorname{Alt}\left(\varphi_{1} \rightarrow \varphi_{2}\right)$, we have $s^{\perp} \in \operatorname{Alt}\left(\varphi_{1} \rightarrow \varphi_{2}\right)$ and $l(s) \leq k$.

- If $l(s)<k$ for all $s \in A l t\left(\varphi_{1}\right)$, then $\varphi_{1}$ is in $\bigcup_{i<k} \mathrm{~F}_{i} \subseteq \mathrm{E}_{k}^{+}$.

- Otherwise, there is $s_{0} \in \operatorname{Alt}\left(\varphi_{1}\right)$ such that $l\left(s_{0}\right)=k$. Since $s_{0}^{\perp} \in$ $\operatorname{Alt}\left(\varphi_{1} \rightarrow \varphi_{2}\right)$, we have $\varphi_{1} \rightarrow \varphi_{2} \in \mathrm{U}_{k}$. Then, for each $s \in \operatorname{Alt}\left(\varphi_{1}\right)$ such that $l(s)=k$, we have $i\left(s^{\perp}\right) \equiv-$, and hence, $i(s) \equiv+$. Thus $\varphi_{1} \in \mathrm{E}_{k} \subseteq \mathrm{E}_{k}^{+}$.

We also have $\varphi_{2} \in \mathrm{U}_{k}^{+}$in the same manner.

For the converse direction, assume $\varphi_{1} \in \mathrm{E}_{k}^{+}$and $\varphi_{2} \in \mathrm{U}_{k}^{+}$. Since $\operatorname{deg}\left(\varphi_{1}\right) \leq k$ and $\operatorname{deg}\left(\varphi_{2}\right) \leq k$, we have $\operatorname{deg}\left(\varphi_{1} \rightarrow \varphi_{2}\right) \leq k$.

- If $\operatorname{deg}\left(\varphi_{1} \rightarrow \varphi_{2}\right)<k$, then $\varphi_{1} \rightarrow \varphi_{2} \in \bigcup_{i<k} \mathrm{~F}_{i} \subseteq \mathrm{U}_{k}^{+}$.

- If $\operatorname{deg}\left(\varphi_{1} \rightarrow \varphi_{2}\right)=k$, for all $s \in \operatorname{Alt}\left(\varphi_{1} \rightarrow \varphi_{2}\right)$ such that $l(s)=k$, we have $s \in \operatorname{Alt}\left(\varphi_{2}\right)$ or $s \equiv s_{0}{ }^{\perp}$ for some $s_{0} \in \operatorname{Alt}\left(\varphi_{1}\right)$. In the former case, we have $i(s) \equiv-$ by $\varphi_{2} \in \mathrm{U}_{k}^{+}$. In the latter case, we have $i\left(s_{0}\right) \equiv+$ by $\varphi_{1} \in \mathrm{E}_{k}^{+}$, and hence, $i(s) \equiv-$.

One can also show that $\varphi_{1} \rightarrow \varphi_{2}$ is in $\mathrm{E}_{k}^{+}$if and only if $\varphi_{1}$ is in $\mathrm{U}_{k}^{+}$and $\varphi_{2}$ is in $\mathrm{E}_{k}^{+}$analogously.

(4): Assume $\forall x \varphi_{1} \in \mathrm{U}_{k}^{+}$.

- If $\forall x \varphi_{1} \notin \mathrm{U}_{k}$, then $\forall x \varphi_{1} \in \bigcup_{i<k} \mathrm{~F}_{i}$. Since $\operatorname{deg}\left(\varphi_{1}\right) \leq \operatorname{deg}\left(\forall x \varphi_{1}\right)<k$, we have $\varphi_{1} \in \bigcup_{i<k} \mathrm{~F}_{i} \subseteq \mathrm{U}_{k}^{+}$.

- Otherwise, $\operatorname{deg}\left(\varphi_{1}\right) \leq \operatorname{deg}\left(\forall x \varphi_{1}\right)=k$. If $\operatorname{deg}\left(\varphi_{1}\right)<k$, then we have $\varphi_{1} \in \bigcup_{i<k} \mathrm{~F}_{i} \subseteq \mathrm{U}_{k}^{+}$. Assume $\operatorname{deg}\left(\varphi_{1}\right)=k$. Let $s$ be an alternation path of $\varphi_{1}$ such that $l(s)=k$. If $i(s) \not \equiv-$, by the definition of $\operatorname{Alt}\left(\forall x \varphi_{1}\right)$, we have $-s \in \operatorname{Alt}\left(\forall x \varphi_{1}\right)$, which contradicts $\operatorname{deg}\left(\forall x \varphi_{1}\right)=k$ since $l(-s)=$ $k+1$. Then we have $i(s) \equiv-$. Thus we have $\varphi_{1} \in \mathrm{U}_{k} \subseteq \mathrm{U}_{k}^{+}$.

For the converse direction, assume $\varphi_{1} \in \mathrm{U}_{k}^{+}$. 
- If $\varphi_{1} \notin \mathrm{U}_{k}$, then $\varphi_{1} \in \bigcup_{i<k} \mathrm{~F}_{i}$. Thus $\operatorname{deg}\left(\varphi_{1}\right)<k$, and hence, $\operatorname{deg}\left(\forall x \varphi_{1}\right) \leq$ $k$. If $\operatorname{deg}\left(\forall x \varphi_{1}\right)<k$, then $\forall x \varphi_{1} \in \bigcup_{i<k} \mathrm{~F}_{i} \subseteq \mathrm{U}_{k}^{+}$. If $\operatorname{deg}\left(\forall x \varphi_{1}\right)=k$, since $i(s) \equiv-$ for all $s \in \operatorname{Alt}\left(\forall x \varphi_{1}\right)$, we have $\forall x \varphi_{1} \in \mathrm{U}_{k} \subseteq \mathrm{U}_{k}^{+}$.

- Otherwise, $\operatorname{deg}\left(\varphi_{1}\right)=k$ and $i(s) \equiv-$ for all $s \in \operatorname{Alt}\left(\varphi_{1}\right)$ such that $l(s)=k$. By the definition of $\operatorname{Alt}\left(\forall x \varphi_{1}\right)$, for all $s \in \operatorname{Alt}\left(\forall x \varphi_{1}\right)$, we have $l(s) \leq k$, and hence, $\operatorname{deg}\left(\forall x \varphi_{1}\right)=k$. In addition, again by the definition of $\operatorname{Alt}\left(\forall x \varphi_{1}\right)$, we have $i(s)=-$ for all $s \in A l t\left(\forall x \varphi_{1}\right)$ such that $l(s)=k$. Thus $\forall x \varphi_{1} \in \mathrm{U}_{k} \subseteq \mathrm{U}_{k}^{+}$.

(5): Analogous to (4).

(6): Assume $\forall x \varphi_{1} \in \mathrm{E}_{k+1}^{+}$. Since $i(s) \equiv-$ for all $s \in \operatorname{Alt}\left(\forall x \varphi_{1}\right), \forall x \varphi_{1}$ is not in $\mathrm{E}_{k+1}$. Then $\forall x \varphi_{1} \in \bigcup_{i \leq k} \mathrm{~F}_{i}$, and hence, $\operatorname{deg}\left(\forall x \varphi_{1}\right) \leq k$.

- If $\operatorname{deg}\left(\forall x \varphi_{1}\right)<k$, then $\forall x \varphi_{1} \in \bigcup_{i<k} \mathrm{~F}_{i} \subseteq \mathrm{U}_{k}^{+}$.

- If $\operatorname{deg}\left(\forall x \varphi_{1}\right)=k$, since $i(s) \equiv-$ for all $s \in A l t\left(\forall x \varphi_{1}\right)$, we have $\forall x \varphi_{1} \in \mathrm{U}_{k} \subseteq \mathrm{U}_{k}^{+}$.

The converse direction is trivial since $\mathrm{U}_{k}^{+} \subseteq \bigcup_{i<k+1} \mathrm{~F}_{i} \subseteq \mathrm{E}_{k+1}^{+}$.

(7): Analogous to (므).

Lemma 4.6. Let $k$ be a natural number. For all $\varphi_{1}$ and $\varphi_{2}$ in $\Pi_{k}$, there exists $\varphi \in \Pi_{k}$ such that $\mathrm{FV}(\varphi)=\mathrm{FV}\left(\varphi_{1}\right) \cup \mathrm{FV}\left(\varphi_{2}\right)$ and $\mathrm{HA}+\neg \neg \Sigma_{k-1}$-DNE (HA if $k=0)$ proves $\neg \neg\left(\varphi_{1} \vee \varphi_{2}\right) \leftrightarrow \neg \neg \varphi$.

Proof. Without loss of generality, assume $k>0, \varphi_{1}: \equiv \forall x \rho_{1}(x)$ and $\varphi_{2}: \equiv$ $\forall y \rho_{2}(y)$ where $\rho_{1}(x), \rho_{2}(y) \in \Sigma_{k-1}$ (see Remark 2.2). By Lemma 4.4, it suffices to show

$\mathrm{HA}+\neg \neg \Sigma_{k-1}$-DNE $\vdash \neg \neg\left(\forall x \rho_{1}(x) \vee \forall y \rho_{2}(y)\right) \leftrightarrow \neg \neg \forall x, y\left(\rho_{1}(x) \vee \rho_{2}(y)\right)$.

The implication from the left to the right is straightforward. The converse implication is shown as follows:

$$
\begin{array}{rlrl} 
& \neg \neg \forall x, y\left(\rho_{1}(x) \vee \rho_{2}(y)\right) \\
\neg \neg \overleftrightarrow{\Sigma_{k-1} \text {-DNE }} & \neg \neg \forall x, y\left(\neg \neg \rho_{1}(x) \vee \neg \neg \rho_{2}(y)\right) \\
& \longleftrightarrow & \forall x, y \neg \neg\left(\neg \neg \rho_{1}(x) \vee \neg \neg \rho_{2}(y)\right) \\
& \longleftrightarrow & \neg \exists x, y \neg\left(\neg \neg \rho_{1}(x) \vee \neg \neg \rho_{2}(y)\right) \\
& \longleftrightarrow \quad \neg \exists x, y\left(\neg \rho_{1}(x) \wedge \neg \rho_{2}(y)\right) \\
& \longleftrightarrow \quad \neg\left(\neg \neg \exists x \neg \rho_{1}(x) \wedge \neg \neg \exists y \neg \rho_{2}(y)\right) \\
& \longleftrightarrow \quad \neg\left(\neg \forall x \neg \neg \rho_{1}(x) \wedge \neg \forall y \neg \neg \rho_{2}(y)\right) \\
\neg \neg \Sigma_{k-1-\mathrm{DNE}} & \neg \neg\left(\forall x \rho_{1}(x) \vee \forall y \rho_{2}(y)\right) .
\end{array}
$$


Lemma 4.7. Let $k$ be a natural number.

1. For all $\varphi \in \Pi_{k}$, there exists $\psi \in \Sigma_{k}$ such that $\mathrm{FV}(\varphi)=\mathrm{FV}(\psi)$ and $\mathrm{HA}+\Sigma_{k}$-DNE proves $\neg \varphi \leftrightarrow \psi$.

2. For all $\varphi \in \Sigma_{k}$, there exists $\psi \in \Pi_{k}$ such that $\mathrm{FV}(\varphi)=\mathrm{FV}(\psi)$ and $\mathrm{HA}+\Sigma_{k-1}$-DNE (HA if $\left.k=0\right)$ proves $\neg \varphi \leftrightarrow \psi$.

Proof. By simultaneous induction on $k$. The base case is trivial. In what follows, we show the induction step for $k+1$.

Let $\varphi: \equiv \forall x \rho(x)$ where $\rho(x) \in \Sigma_{k}$. By induction hypothesis, there exists $\rho^{\prime}(x) \in \Pi_{k}$ such that $\mathrm{FV}(\rho(x))=\mathrm{FV}\left(\rho^{\prime}(x)\right)$ and

$$
\mathrm{HA}+\Sigma_{k-1}-\mathrm{DNE} \vdash \neg \rho(x) \leftrightarrow \rho^{\prime}(x) .
$$

Then HA $+\Sigma_{k+1}$-DNE proves

$$
\begin{aligned}
& \begin{array}{ll} 
& \neg \forall x \rho(x) \\
\Sigma_{k \text {-DNE }} & \neg \forall x \neg \neg \rho(x) \\
\longleftrightarrow & \neg \neg \exists x \neg \rho(x) \\
\overleftrightarrow{\longleftrightarrow} & \neg \neg \exists x \rho^{\prime}(x)
\end{array} \\
& \underset{\Sigma_{k+1} \text {-DNE }}{\overleftrightarrow{\rightleftarrows}} \exists x \rho^{\prime}(x)
\end{aligned}
$$

which is in $\Sigma_{k+1}$.

Next, let $\varphi: \equiv \exists x \rho(x)$ where $\rho(x) \in \Pi_{k}$. By induction hypothesis, there exists $\rho^{\prime}(x) \in \Sigma_{k}$ such that $\mathrm{FV}(\rho(x))=\mathrm{FV}\left(\rho^{\prime}(x)\right)$ and

$$
\mathrm{HA}+\Sigma_{k} \text {-DNE } \vdash \neg \rho(x) \leftrightarrow \rho^{\prime}(x) .
$$

Then HA $+\Sigma_{k}$-DNE proves

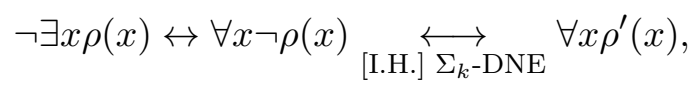

which is in $\Pi_{k+1}$.

Lemma 4.8. Let $k$ be a natural number. For all $\varphi \in \Pi_{k}$, there exists $\psi \in \Sigma_{k}$ such that $\mathrm{FV}(\varphi)=\mathrm{FV}(\psi)$ and $\mathrm{HA}+\neg \neg \Sigma_{k}-\mathrm{DNE} \vdash \neg \varphi \leftrightarrow \neg \neg \psi$.

Proof. Let $\varphi \in \Pi_{k}$. By Lemma 4.7,(1), there exists $\psi \in \Sigma_{k}$ such that $\mathrm{FV}(\varphi)=\mathrm{FV}(\psi)$ and $\mathrm{HA}+\Sigma_{k}$-DNE $\vdash \neg \varphi \leftrightarrow \psi$. By Corollary 4.2, we have $\mathrm{HA}+\neg \neg \Sigma_{k}$-DNE $\vdash \neg \varphi \leftrightarrow \neg \neg \psi$.

Lemma 4.9. $\mathrm{HA}+\mathrm{U}_{k}^{+}$-DNS $\vdash \neg \neg \Sigma_{k-1}$-LEM for each natural number $k>0$. 
Proof. Fix an instance of $\neg \neg \Sigma_{k-1}$-LEM

$$
\varphi: \equiv \neg \neg \forall x\left(\varphi_{1}(x) \vee \neg \varphi_{1}(x)\right),
$$

where $\varphi_{1}(x) \in \Sigma_{k-1}$. Note $\left(\varphi_{1}(x) \vee \neg \varphi_{1}(x)\right) \in \mathrm{F}_{k-1} \subseteq \mathrm{U}_{k}^{+}$. Since HA proves $\forall x \neg \neg\left(\varphi_{1}(x) \vee \neg \varphi_{1}(x)\right)$, we have that $\mathrm{HA}+\mathrm{U}_{k}^{+}$-DNS proves $\neg \neg \forall x\left(\varphi_{1}(x) \vee \neg \varphi_{1}(x)\right)$, namely, $\varphi$.

Corollary 4.10. $\mathrm{HA}+\mathrm{U}_{k}^{+}$-DNS $\vdash \neg \neg \Sigma_{k-1}$-DNE for each natural number $k>0$.

Proof. Immediate from Remark 2.8, Lemma 4.9 and the fact that $\Sigma_{k-1}$-LEM implies $\Sigma_{k-1}$-DNE.

Remark 4.11. By Remark 2.12, $\mathrm{U}_{k}^{+}$-DNS is equivalent to $\mathrm{U}_{k}$-DNS over HA. Then $\mathrm{U}_{k}^{+}$-DNS can be replaced by $\mathrm{U}_{k}$-DNS throughout the paper.

\section{Prenex normal form theorems}

In this section, we show the modified version of [1, Theorem 2.7]. Prior to that, we first show a variant of the prenex normal form theorem:

Lemma 5.1. For each natural number $k$ and a formula $\varphi$ (possibly containing free variables), if $\varphi \in \mathrm{U}_{k}^{+}$, then there exists $\varphi^{\prime} \in \Pi_{k}$ such that $\mathrm{FV}(\varphi)=\mathrm{FV}\left(\varphi^{\prime}\right)$ and

$$
\mathrm{HA}+\mathrm{U}_{k}^{+}-\mathrm{DNS} \vdash \neg \neg \varphi \leftrightarrow \neg \neg \varphi^{\prime} .
$$

Proof. By simultaneous induction on $k$, we show the following two statements (which are in fact equivalent):

1. if $\varphi \in \mathrm{E}_{k}^{+}$, then there exists $\varphi^{\prime} \in \Pi_{k}$ such that $\mathrm{FV}(\varphi)=\mathrm{FV}\left(\varphi^{\prime}\right)$ and

$$
\mathrm{HA}+\mathrm{U}_{k}^{+}-\mathrm{DNS} \vdash \neg \varphi \leftrightarrow \neg \neg \varphi^{\prime} ;
$$

2. if $\varphi \in \mathrm{U}_{k}^{+}$, then there exists $\varphi^{\prime} \in \Pi_{k}$ such that $\mathrm{FV}(\varphi)=\mathrm{FV}\left(\varphi^{\prime}\right)$ and

$$
\mathrm{HA}+\mathrm{U}_{k}^{+}-\mathrm{DNS} \vdash \neg \neg \varphi \leftrightarrow \neg \neg \varphi^{\prime} .
$$

The base case is trivial (one can take $\varphi^{\prime}$ as $\varphi$ itself). In what follows, we show the induction step.

For the induction step, assume the items 1 and 2 for $k-1$. We show the items 1 and 2 for $k$ simultaneously by induction on the structure of formulas. When $\varphi$ is a prime formula, by Lemma 2.3, we have $\varphi^{\prime}$ which satisfies the requirement. For the induction step, assume that the items 1 and 2 hold for $\varphi_{1}$ and $\varphi_{2}$. When it is clear from the context, we suppress the argument on free variables. 
The case of $\varphi_{1} \wedge \varphi_{2}$ : First, assume $\varphi_{1} \wedge \varphi_{2} \in \mathrm{E}_{k}^{+}$. By Lemma 4.5, we have $\varphi_{1}, \varphi_{2} \in \mathrm{E}_{k}^{+}$. By induction hypothesis, there exist $\varphi_{1}^{\prime}, \varphi_{2}^{\prime} \in \Pi_{k}$ such that $\mathrm{HA}+\mathrm{U}_{k}^{+}$-DNS $\vdash\left(\neg \varphi_{1} \leftrightarrow \neg \neg \varphi_{1}^{\prime}\right) \wedge\left(\neg \varphi_{2} \leftrightarrow \neg \neg \varphi_{2}^{\prime}\right)$. By Lemma 4.6, there exists $\varphi^{\prime} \in \Pi_{k}$ such that $\mathrm{FV}\left(\varphi^{\prime}\right)=\mathrm{FV}\left(\varphi_{1}^{\prime}\right) \cup \mathrm{FV}\left(\varphi_{2}^{\prime}\right)$ and

$$
\mathrm{HA}+\neg \neg \Sigma_{k-1} \text {-DNE } \vdash \neg \neg\left(\varphi_{1}^{\prime} \vee \varphi_{2}^{\prime}\right) \leftrightarrow \neg \neg \varphi^{\prime} .
$$

By Corollary 4.10, $\mathrm{HA}+\mathrm{U}_{k}^{+}$-DNS proves

$$
\begin{aligned}
& \neg\left(\varphi_{1} \wedge \varphi_{2}\right) \\
\longleftrightarrow & \neg\left(\neg \neg \varphi_{1} \wedge \neg \neg \varphi_{2}\right) \\
\text { [I.H.] } \mathrm{U}_{k}^{+} \text {-DNS } & \neg\left(\neg \varphi_{1}^{\prime} \wedge \neg \varphi_{2}^{\prime}\right) \\
\longleftrightarrow & \neg \neg\left(\varphi_{1}^{\prime} \vee \neg \varphi_{2}^{\prime}\right) \\
\stackrel{\longleftrightarrow}{\longleftrightarrow} & \neg \neg \varphi^{\prime} .
\end{aligned}
$$

Next, assume $\varphi_{1} \wedge \varphi_{2} \in \mathrm{U}_{k}^{+}$. By Lemma 4.5, we have $\varphi_{1}, \varphi_{2} \in \mathrm{U}_{k}^{+}$. By induction hypothesis, there exist $\varphi_{1}^{\prime}, \varphi_{2}^{\prime} \in \Pi_{k}$ such that $\mathrm{HA}+\mathrm{U}_{k}^{+}$-DNS $\vdash$ $\left(\neg \neg \varphi_{1} \leftrightarrow \neg \neg \varphi_{1}^{\prime}\right) \wedge\left(\neg \neg \varphi_{2} \leftrightarrow \neg \neg \varphi_{2}^{\prime}\right)$. By Lemma 4.3, there exists $\varphi^{\prime} \in \Pi_{k}$ such that $\mathrm{FV}\left(\varphi^{\prime}\right)=\mathrm{FV}\left(\varphi_{1}^{\prime}\right) \cup \mathrm{FV}\left(\varphi_{2}^{\prime}\right)$ and $\mathrm{HA} \vdash \varphi^{\prime} \leftrightarrow \varphi_{1}^{\prime} \wedge \varphi_{2}^{\prime}$. Then $\mathrm{HA}+\mathrm{U}_{k}^{+}$-DNS proves

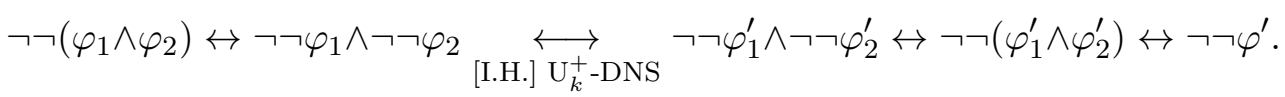

The case of $\varphi_{1} \vee \varphi_{2}$ : First, assume $\varphi_{1} \vee \varphi_{2} \in \mathrm{E}_{k}^{+}$. By Lemma 4.5, we have $\varphi_{1}, \varphi_{2} \in \mathrm{E}_{k}^{+}$. By induction hypothesis, there exist $\varphi_{1}^{\prime}, \varphi_{2}^{\prime} \in \Pi_{k}$ such that $\mathrm{HA}+\mathrm{U}_{k}^{+}$-DNS $\vdash\left(\neg \varphi_{1} \leftrightarrow \neg \neg \varphi_{1}^{\prime}\right) \wedge\left(\neg \varphi_{2} \leftrightarrow \neg \neg \varphi_{2}^{\prime}\right)$. By Lemma 4.3, there exists $\varphi^{\prime} \in \Pi_{k}$ such that $\mathrm{FV}\left(\varphi^{\prime}\right)=\mathrm{FV}\left(\varphi_{1}^{\prime}\right) \cup \mathrm{FV}\left(\varphi_{2}^{\prime}\right)$ and $\mathrm{HA} \vdash \varphi^{\prime} \leftrightarrow \varphi_{1}^{\prime} \wedge \varphi_{2}^{\prime}$. Then $\mathrm{HA}+\mathrm{U}_{k}^{+}$-DNS proves

$$
\begin{array}{cll} 
& \neg\left(\varphi_{1} \vee \varphi_{2}\right) \\
\longleftrightarrow & \neg \varphi_{1} \wedge \neg \varphi_{2} \\
\text { [I.H.] } \mathrm{U}_{k}^{+} \text {-DNS } & \neg \neg \varphi_{1}^{\prime} \wedge \neg \neg \varphi_{2}^{\prime} \\
\longleftrightarrow & \neg \neg\left(\varphi_{1}^{\prime} \wedge \varphi_{2}^{\prime}\right) \\
\longleftrightarrow & \neg \neg \varphi^{\prime} .
\end{array}
$$

Next, assume $\varphi_{1} \vee \varphi_{2} \in \mathrm{U}_{k}^{+}$. By Lemma 4.5, we have $\varphi_{1}, \varphi_{2} \in \mathrm{U}_{k}^{+}$. By induction hypothesis, there exist $\varphi_{1}^{\prime}, \varphi_{2}^{\prime} \in \Pi_{k}$ such that $\mathrm{HA}+\mathrm{U}_{k}^{+}$-DNS $\vdash$ $\left(\neg \neg \varphi_{1} \leftrightarrow \neg \neg \varphi_{1}^{\prime}\right) \wedge\left(\neg \neg \varphi_{2} \leftrightarrow \neg \neg \varphi_{2}^{\prime}\right)$. By Lemma 4.6, there exists $\varphi^{\prime} \in \Pi_{k}$ such that $\mathrm{FV}\left(\varphi^{\prime}\right)=\mathrm{FV}\left(\varphi_{1}^{\prime}\right) \cup \mathrm{FV}\left(\varphi_{2}^{\prime}\right)$ and

$$
\mathrm{HA}+\neg \neg \Sigma_{k-1}-\mathrm{DNE} \vdash \neg \neg\left(\varphi_{1}^{\prime} \vee \varphi_{2}^{\prime}\right) \leftrightarrow \neg \neg \varphi^{\prime} .
$$


By Corollary 4.10, $\mathrm{HA}+\mathrm{U}_{k}^{+}$-DNS proves

$$
\begin{array}{cl} 
& \neg \neg\left(\varphi_{1} \vee \varphi_{2}\right) \\
\longleftrightarrow & \neg \neg\left(\neg \neg \varphi_{1} \vee \neg \neg \varphi_{2}\right) \\
\text { [I.H.] } \mathrm{U}_{k}^{+} \text {-DNS } & \neg \neg\left(\neg \neg \varphi_{1}^{\prime} \vee \neg \neg \varphi_{2}^{\prime}\right) \\
\longleftrightarrow & \neg \neg\left(\varphi_{1}^{\prime} \vee \varphi_{2}^{\prime}\right) \\
\neg \neg \Sigma_{k-1} \text {-DNE } & \neg \neg \varphi^{\prime} .
\end{array}
$$

The case of $\varphi_{1} \rightarrow \varphi_{2}$ : First, assume $\varphi_{1} \rightarrow \varphi_{2} \in \mathrm{E}_{k}^{+}$. By Lemma 4.5, we have $\varphi_{1} \in \mathrm{U}_{k}^{+}$and $\varphi_{2} \in \mathrm{E}_{k}^{+}$. By induction hypothesis, there exist $\varphi_{1}^{\prime}, \varphi_{2}^{\prime} \in$ $\Pi_{k}$ such that $\mathrm{HA}+\mathrm{U}_{k}^{+}$-DNS proves $\neg \neg \varphi_{1} \leftrightarrow \neg \neg \varphi_{1}^{\prime}$ and $\neg \varphi_{2} \leftrightarrow \neg \neg \varphi_{2}^{\prime}$. By Lemma 4.3, there exists $\varphi^{\prime} \in \Pi_{k}$ such that $\mathrm{FV}\left(\varphi^{\prime}\right)=\mathrm{FV}\left(\varphi_{1}^{\prime}\right) \cup \mathrm{FV}\left(\varphi_{2}^{\prime}\right)$ and $\mathrm{HA} \vdash \varphi^{\prime} \leftrightarrow \varphi_{1}^{\prime} \wedge \varphi_{2}^{\prime}$. Then HA $+\mathrm{U}_{k}^{+}$-DNS proves

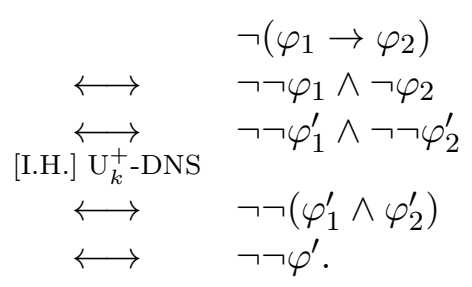

Next, assume $\varphi_{1} \rightarrow \varphi_{2} \in \mathrm{U}_{k}^{+}$. By Lemma 4.5, we have $\varphi_{1} \in \mathrm{E}_{k}^{+}$and $\varphi_{2} \in \mathrm{U}_{k}^{+}$. By induction hypothesis, there exist $\varphi_{1}^{\prime}, \varphi_{2}^{\prime} \in \Pi_{k}$ such that $\mathrm{HA}+\mathrm{U}_{k}^{+}$-DNS proves $\neg \varphi_{1} \leftrightarrow \neg \neg \varphi_{1}^{\prime}$ and $\neg \neg \varphi_{2} \leftrightarrow \neg \neg \varphi_{2}^{\prime}$. By Lemma 4.6, there exists $\varphi^{\prime} \in \Pi_{k}$ such that $\mathrm{FV}\left(\varphi^{\prime}\right)=\mathrm{FV}\left(\varphi_{1}^{\prime}\right) \cup \mathrm{FV}\left(\varphi_{2}^{\prime}\right)$ and

$$
\mathrm{HA}+\neg \neg \Sigma_{k-1} \text {-DNE } \vdash \neg \neg\left(\varphi_{1}^{\prime} \vee \varphi_{2}^{\prime}\right) \leftrightarrow \neg \neg \varphi^{\prime} .
$$

By Corollary 4.10, $\mathrm{HA}+\mathrm{U}_{k}^{+}$-DNS proves

$$
\begin{array}{cl} 
& \neg \neg\left(\varphi_{1} \rightarrow \varphi_{2}\right) \\
\longleftrightarrow & \neg\left(\neg \neg \varphi_{1} \wedge \neg \varphi_{2}\right) \\
\text { [I.H.] } \mathrm{U}_{k}^{+} \text {-DNS } & \neg\left(\neg \varphi_{1}^{\prime} \wedge \neg \varphi_{2}^{\prime}\right) \\
\longleftrightarrow & \neg \neg\left(\varphi_{1}^{\prime} \vee \varphi_{2}^{\prime}\right) \\
\neg \neg \Sigma_{k-1} \text {-DNE } & \neg \neg \varphi^{\prime} .
\end{array}
$$

The case of $\forall x \varphi_{1}(x)$ : First, assume $\forall x \varphi_{1}(x) \in \mathrm{E}_{k}^{+}$. By Lemma 4.5, we have $\forall x \varphi_{1}(x) \in \mathrm{U}_{k-1}^{+}$. By the item 2 for $k-1$, there exists $\varphi^{\prime} \in \Pi_{k-1}$ such that

$$
\mathrm{HA}+\mathrm{U}_{k-1}^{+} \text {-DNS } \vdash \neg \neg \forall x \varphi_{1}(x) \leftrightarrow \neg \neg \varphi^{\prime} .
$$

By Lemma 4.8, there exists $\varphi^{\prime \prime} \in \Sigma_{k-1} \subseteq \Pi_{k}$ (see Remark 2.5) such that $\mathrm{FV}\left(\varphi^{\prime}\right)=\mathrm{FV}\left(\varphi^{\prime \prime}\right)$ and

$$
\mathrm{HA}+\neg \neg \Sigma_{k-1}-\mathrm{DNE} \vdash \neg \varphi^{\prime} \leftrightarrow \neg \neg \varphi^{\prime \prime} .
$$


By Corollary 4.10, $\mathrm{HA}+\mathrm{U}_{k}^{+}$-DNS proves

$$
\neg \forall x \varphi_{1}(x) \underset{\text { [I.H.] } \mathrm{U}_{k-1}^{+} \text {-DNS }}{\longleftrightarrow} \neg \varphi^{\prime} \underset{\neg \neg \Sigma_{k-1} \text {-DNE }}{\longleftrightarrow} \neg \neg \varphi^{\prime \prime} .
$$

Next, assume $\forall x \varphi_{1}(x) \in \mathrm{U}_{k}^{+}$. By Lemma 4.5, we have $\varphi_{1}(x) \in \mathrm{U}_{k}^{+}$. By induction hypothesis, there exists $\varphi_{1}^{\prime}(x) \in \Pi_{k}$ such that

$$
\mathrm{HA}+\mathrm{U}_{k}^{+} \text {-DNS } \vdash \neg \neg \varphi_{1}(x) \leftrightarrow \neg \neg \varphi_{1}^{\prime}(x) .
$$

Then $\mathrm{HA}+\mathrm{U}_{k}^{+}$-DNS proves

$$
\neg \neg \forall x \varphi_{1}(x) \underset{\mathrm{U}_{k}^{+} \text {-DNS }}{\longleftrightarrow} \forall x \neg \neg \varphi_{1}(x) \underset{\text { [I.H.] } \mathrm{U}_{k}^{+} \text {-DNS }}{\longleftrightarrow} \forall x \neg \neg \varphi_{1}^{\prime}(x) \underset{\mathrm{U}_{k}^{+} \text {-DNS }}{\longleftrightarrow} \neg \neg \forall x \varphi_{1}^{\prime}(x)
$$

The case of $\exists x \varphi_{1}(x)$ : First, assume $\exists x \varphi_{1}(x) \in \mathrm{E}_{k}^{+}$. By Lemma 4.5, we have $\varphi_{1}(x) \in \mathrm{E}_{k}^{+}$. By induction hypothesis, there exists $\varphi_{1}^{\prime}(x) \in \Pi_{k}$ such that

$$
\mathrm{HA}+\mathrm{U}_{k}^{+} \text {-DNS } \vdash \neg \varphi_{1}(x) \leftrightarrow \neg \neg \varphi_{1}^{\prime}(x) .
$$

Then $\mathrm{HA}+\mathrm{U}_{k}^{+}$-DNS proves

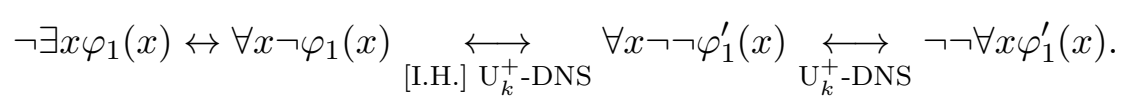

Next, assume that $\exists x \varphi_{1}(x) \in \mathrm{U}_{k}^{+}$. By Lemma 4.5, we have $\exists x \varphi_{1}(x) \in$ $\mathrm{E}_{k-1}^{+}$. By the item 1 for $k-1$, there exists $\varphi^{\prime} \in \Pi_{k-1}$ such that

$$
\mathrm{HA}+\mathrm{U}_{k-1}^{+} \text {-DNS } \vdash \neg \exists x \varphi_{1}(x) \leftrightarrow \neg \neg \varphi^{\prime} .
$$

By Lemma 4.8, there exists $\varphi^{\prime \prime} \in \Sigma_{k-1} \subseteq \Pi_{k}$ (see Remark 2.5) such that $\mathrm{FV}\left(\varphi^{\prime}\right)=\mathrm{FV}\left(\varphi^{\prime \prime}\right)$ and

$$
\mathrm{HA}+\neg \neg \Sigma_{k-1}-\mathrm{DNE} \vdash \neg \varphi^{\prime} \leftrightarrow \neg \neg \varphi^{\prime \prime} .
$$

By Corollary 4.10, $\mathrm{HA}+\mathrm{U}_{k}^{+}$-DNS proves

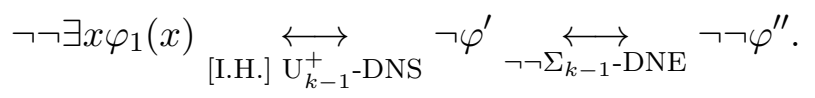

The following lemma is used a lot of times implicitly in the proof of our prenex normal form theorem (Theorem 5.3).

Lemma 5.2 (cf. Fact 2.2 in [1]). Let $k$ be a natural number.

1. $\mathrm{HA}+\Sigma_{k+1}$-DNE $\vdash\left(\Pi_{k} \vee \Pi_{k}\right)$-DNE.

2. $\mathrm{HA}+\left(\Pi_{k+1} \vee \Pi_{k+1}\right)-\mathrm{DNE} \vdash \Sigma_{k}$-DNE. 
3. $\mathrm{HA}+\neg \neg\left(\Pi_{k+1} \vee \Pi_{k+1}\right)-\mathrm{DNE} \vdash \neg \neg \Sigma_{k}$-DNE.

4. $\mathrm{HA}+\Sigma_{k}$-DNE $\vdash \Pi_{k+1}$-DNE.

Proof. (11): For formulas $\varphi_{1}$ and $\varphi_{2}$ in $\Pi_{k}, \varphi_{1} \vee \varphi_{2}$ is equivalent (over HA) to

$$
\exists k\left(\left(k=0 \rightarrow \varphi_{1}\right) \wedge\left(k \neq 0 \rightarrow \varphi_{2}\right)\right),
$$

which is equivalent to some $\varphi \in \Sigma_{k+1}$ such that $\mathrm{FV}(\varphi)=\mathrm{FV}\left(\varphi_{1} \vee \varphi_{2}\right)$ over HA by Lemma 4.3. (2). Therefore any instance of $\left(\Pi_{k} \vee \Pi_{k}\right)$-DNE is derived from some instance of $\Sigma_{k+1}$-DNE.

(2): Any instance of $\Sigma_{k}$-DNE is derived from some instance of $\left(\Pi_{k+1} \vee \Pi_{k+1}\right)$-DNE since $\varphi \in \Sigma_{k}$ is equivalent to $\forall y \varphi \in \Pi_{k+1}$ with a variable $y$ not occurring freely in $\varphi$ (cf. Lemma 2.3).

(3): Immediate from (2) and Corollary 4.2.

(44): Note that $\neg \neg \forall x \varphi(x)$ implies $\neg \neg \forall x \neg \neg \varphi(x)$, which is intuitionistically equivalent to $\forall x \neg \neg \varphi(x)$. Then any instance of $\Pi_{k+1}$-DNE is derived from some instance of $\Sigma_{k}$-DNE.

We are now ready to show the modified version of [1, Theorem 2.7].

Theorem 5.3. For each natural number $k$ and a formula $\varphi$ (possibly containing free variables), the following hold:

1. if $\varphi \in \mathrm{E}_{k}^{+}$, then there exists $\varphi^{\prime} \in \Sigma_{k}$ such that $\mathrm{FV}(\varphi)=\mathrm{FV}\left(\varphi^{\prime}\right)$ and

$$
\mathrm{HA}+\Sigma_{k}-\mathrm{DNE}+\mathrm{U}_{k}^{+}-\mathrm{DNS} \vdash \varphi \leftrightarrow \varphi^{\prime} ;
$$

2. if $\varphi \in \mathrm{U}_{k}^{+}$, then there exists $\varphi^{\prime} \in \Pi_{k}$ such that $\mathrm{FV}(\varphi)=\mathrm{FV}\left(\varphi^{\prime}\right)$ and

$$
\mathrm{HA}+\left(\Pi_{k} \vee \Pi_{k}\right)-\mathrm{DNE} \vdash \varphi \leftrightarrow \varphi^{\prime} .
$$

Proof. For the proof, we prepare the following auxiliary assertion (which is in fact a consequence from the item 21):

3. if $\varphi \in \mathrm{E}_{k}^{+}$, then there exists $\varphi^{\prime} \in \Pi_{k}$ such that $\mathrm{FV}(\varphi)=\mathrm{FV}\left(\varphi^{\prime}\right)$ and

$$
\mathrm{HA}+\neg \neg\left(\Pi_{k} \vee \Pi_{k}\right)-\mathrm{DNE} \vdash \neg \varphi \leftrightarrow \neg \neg \varphi^{\prime} .
$$

We show the items 1, 22 and 3 by induction on $k$ simultaneously. The base case is trivial (one can take $\varphi^{\prime}$ as $\varphi$ itself). In what follows, we show the induction step.

Assume the items 1, 2 and 3 for $k-1$. Since HA $+\Pi_{k-1}$-DNE $\vdash$ $\Pi_{k-1}$-DNS, by the item 2 for $k-1$, we have

$$
\mathrm{HA}+\left(\Pi_{k-1} \vee \Pi_{k-1}\right)-\mathrm{DNE} \vdash \mathrm{U}_{k-1}^{+} \text {-DNS. }
$$


We show the items 1, 2 and 3 simultaneously by induction on the structure of formulas. When $\varphi$ is a prime formula, by Lemma 2.3, we have $\varphi^{\prime}$ which satisfies the requirement. For the induction step, assume that the items 1, 2 and 3 hold for $\varphi_{1}$ and $\varphi_{2}$. When it is clear from the context, we suppress the argument on free variables.

The case of $\varphi_{1} \wedge \varphi_{2}$ : For the second item, assume $\varphi_{1} \wedge \varphi_{2} \in \mathrm{U}_{k}^{+}$. By Lemma 4.5, we have $\varphi_{1}, \varphi_{2} \in \mathrm{U}_{k}^{+}$. By induction hypothesis, there exist $\varphi_{1}^{\prime}, \varphi_{2}^{\prime} \in \Pi_{k}$ such that $\mathrm{HA}+\left(\Pi_{k} \vee \Pi_{k}\right)$-DNE proves $\varphi_{1} \leftrightarrow \varphi_{1}^{\prime}$ and $\varphi_{2} \leftrightarrow \varphi_{2}^{\prime}$. By Lemma 4.3, there exists $\varphi^{\prime} \in \Pi_{k}$ such that HA $\vdash \varphi^{\prime} \leftrightarrow \varphi_{1}^{\prime} \wedge \varphi_{2}^{\prime}$. Then $\mathrm{HA}+\left(\Pi_{k} \vee \Pi_{k}\right)$-DNE proves

$$
\varphi_{1} \wedge \varphi_{2} \underset{\text { [..H.] }]\left(\Pi_{k} \vee \Pi_{k}\right) \text {-DNE }}{\longleftrightarrow} \varphi_{1}^{\prime} \wedge \varphi_{2}^{\prime} \leftrightarrow \varphi^{\prime}
$$

For the first and third items, assume $\varphi_{1} \wedge \varphi_{2} \in \mathrm{E}_{k}^{+}$. By Lemma 4.5, we have $\varphi_{1}, \varphi_{2} \in \mathrm{E}_{k}^{+}$. Then we have $\varphi^{\prime} \in \Sigma_{k}$ such that $\mathrm{HA}+\Sigma_{k}$-DNE + $\mathrm{U}_{k}^{+}$-DNS $\vdash \varphi_{1} \wedge \varphi_{2} \leftrightarrow \varphi^{\prime}$ as in the second item. On the other hand, by induction hypothesis, there exist $\varphi_{1}^{\prime \prime}, \varphi_{2}^{\prime \prime} \in \Pi_{k}$ such that HA $+\neg \neg\left(\Pi_{k} \vee \Pi_{k}\right)$-DNE proves $\neg \varphi_{1} \leftrightarrow \neg \neg \varphi_{1}^{\prime \prime}$ and $\neg \varphi_{2} \leftrightarrow \neg \neg \varphi_{2}^{\prime \prime}$. In addition, by Lemma 4.6, there exists $\varphi^{\prime \prime} \in \Pi_{k}$ such that HA $+\neg \neg \Sigma_{k-1}$-DNE $\vdash \neg \neg \varphi^{\prime \prime} \leftrightarrow \neg \neg\left(\varphi_{1}^{\prime \prime} \vee \varphi_{2}^{\prime \prime}\right)$. Then, by Lemma 5.2, we have that HA $+\neg \neg\left(\Pi_{k} \vee \Pi_{k}\right)$-DNE proves

$$
\begin{array}{rlrl} 
& \longleftrightarrow & & \neg\left(\varphi_{1} \wedge \varphi_{2}\right) \\
& \longleftrightarrow\left(\neg \neg \varphi_{1} \wedge \neg \neg \varphi_{2}\right) \\
\text { [I.H.] } \neg \neg\left(\Pi_{k} \vee \Pi_{k}\right) \text {-DNE } & \neg \neg\left(\neg \neg \varphi_{1}^{\prime \prime} \vee \neg \neg \varphi_{2}^{\prime \prime}\right) \\
& \longleftrightarrow & & \neg\left(\neg \varphi_{1}^{\prime \prime} \wedge \neg \varphi_{2}^{\prime \prime}\right) \\
& \longleftrightarrow & & \neg \neg\left(\varphi_{1}^{\prime \prime} \vee \varphi_{2}^{\prime \prime}\right) \\
\neg \neg \Sigma_{k-1} \text { DNE } & & \neg \neg \varphi^{\prime \prime} .
\end{array}
$$

The case of $\varphi_{1} \vee \varphi_{2}$ : For the second item, assume $\varphi_{1} \vee \varphi_{2} \in \mathrm{U}_{k}^{+}$. By Lemma 4.5, we have $\varphi_{1}, \varphi_{2} \in \mathrm{U}_{k}^{+}$. Then, by induction hypothesis, there exist $\rho_{1}\left(x_{1}\right), \rho_{2}\left(x_{2}\right) \in \Sigma_{k-1}$ such that $\mathrm{HA}+\left(\Pi_{k} \vee \Pi_{k}\right)$-DNE proves $\varphi_{1} \leftrightarrow$ $\forall x_{1} \rho_{1}\left(x_{1}\right)$ and $\varphi_{2} \leftrightarrow \forall x_{2} \rho_{2}\left(x_{2}\right)$. By Lemma 5.2, $\mathrm{HA}+\left(\Pi_{k} \vee \Pi_{k}\right)$-DNE proves

$$
\begin{aligned}
& \forall x_{1} \rho_{1}\left(x_{1}\right) \vee \forall x_{2} \rho_{2}\left(x_{2}\right) \\
& \longrightarrow \quad \forall x_{1}, x_{2}\left(\rho_{1}\left(x_{1}\right) \vee \rho_{2}\left(x_{2}\right)\right) \\
& \longrightarrow \quad \neg\left(\exists x_{1} \neg \rho_{1}\left(x_{1}\right) \wedge \exists x_{2} \neg \rho_{2}\left(x_{2}\right)\right) \\
& \longleftrightarrow \quad \neg\left(\neg \neg \exists x_{1} \neg \rho_{1}\left(x_{1}\right) \wedge \neg \neg \exists x_{2} \neg \rho_{2}\left(x_{2}\right)\right) \\
& \underset{\Sigma_{k-1} \text {-DNE }}{\overleftrightarrow{\longleftrightarrow}} \neg\left(\neg \forall x_{1} \rho_{1}\left(x_{1}\right) \wedge \neg \forall x_{2} \rho_{2}\left(x_{2}\right)\right) \\
& \longleftrightarrow \quad \neg \neg\left(\forall x_{1} \rho_{1}\left(x_{1}\right) \vee \forall x_{2} \rho_{2}\left(x_{2}\right)\right) \\
& \left(\Pi_{k} \vee \Pi_{k}\right) \text {-DNE } \forall x_{1} \rho_{1}\left(x_{1}\right) \vee \forall x_{2} \rho_{2}\left(x_{2}\right) \text {. }
\end{aligned}
$$


By Lemma 4.4, there exists $\xi\left(x_{1}, x_{2}\right) \in \Sigma_{k-1}$ such that HA $\vdash \xi\left(x_{1}, x_{2}\right) \leftrightarrow$ $\rho_{1}\left(x_{1}\right) \vee \rho_{2}\left(x_{2}\right)$. Then we have that HA $+\left(\Pi_{k} \vee \Pi_{k}\right)$-DNE proves

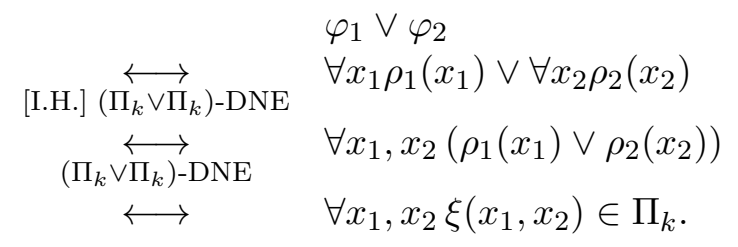

For the first and third items, assume $\varphi_{1} \vee \varphi_{2} \in \mathrm{E}_{k}^{+}$. By Lemma 4.5, we have $\varphi_{1}, \varphi_{2} \in \mathrm{E}_{k}^{+}$. By induction hypothesis, there exist $\rho_{1}\left(x_{1}\right), \rho_{2}\left(x_{2}\right) \in$ $\Pi_{k-1}$ such that $\mathrm{HA}+\Sigma_{k}$-DNE $+\mathrm{U}_{k}^{+}$-DNS proves $\varphi_{1} \leftrightarrow \exists x_{1} \rho_{1}\left(x_{1}\right)$ and $\varphi_{2} \leftrightarrow$ $\exists x_{2} \rho_{2}\left(x_{2}\right)$. By the item 2 for $k-1$, there exists $\xi\left(x_{1}, x_{2}\right) \in \Pi_{k-1}$ such that

$$
\mathrm{HA}+\left(\Pi_{k-1} \vee \Pi_{k-1}\right)-\mathrm{DNE} \vdash \xi\left(x_{1}, x_{2}\right) \leftrightarrow \rho_{1}\left(x_{1}\right) \vee \rho_{2}\left(x_{2}\right)
$$

By Lemma 5.2, we have that $\mathrm{HA}+\Sigma_{k}$-DNE $+\mathrm{U}_{k}^{+}$-DNS proves

$$
\begin{array}{cl} 
& \varphi_{1} \vee \varphi_{2} \\
\text { [I.H.] } \Sigma_{k} \text {-DNE, } \mathrm{U}_{k}^{+} \text {-DNS } & \exists x_{1} \rho_{1}\left(x_{1}\right) \vee \exists x_{2} \rho_{2}\left(x_{2}\right) \\
\stackrel{\longleftrightarrow}{\longleftrightarrow} & \exists x_{1}, x_{2}\left(\rho_{1}\left(x_{1}\right) \vee \rho_{2}\left(x_{2}\right)\right) \\
\text { [I.H.] }\left(\Pi_{k-1} \vee \Pi_{k-1}\right) \text {-DNE } & \exists x_{1}, x_{2} \xi\left(x_{1}, x_{2}\right) .
\end{array}
$$

Thus we are done for the first item. For the third item, by induction hypothesis, there exist $\varphi_{1}^{\prime \prime}, \varphi_{2}^{\prime \prime} \in \Pi_{k}$ such that HA $+\neg \neg\left(\Pi_{k} \vee \Pi_{k}\right)$-DNE proves $\neg \varphi_{1} \leftrightarrow \neg \neg \varphi_{1}^{\prime \prime}$ and $\neg \varphi_{2} \leftrightarrow \neg \neg \varphi_{2}^{\prime \prime}$. In addition, by Lemma 4.3, there exists $\varphi^{\prime \prime} \in \Pi_{k}$ such that HA $\vdash \varphi^{\prime \prime} \leftrightarrow \varphi_{1}^{\prime \prime} \wedge \varphi_{2}^{\prime \prime}$. Then HA $+\neg \neg\left(\Pi_{k} \vee \Pi_{k}\right)$-DNE proves

$\neg\left(\varphi_{1} \vee \varphi_{2}\right) \leftrightarrow \neg \varphi_{1} \wedge \neg \varphi_{2}{ }_{[\mathrm{I} . \mathrm{H} .]} \neg \neg\left(\overleftrightarrow{\left.\Pi_{k} \vee \Pi_{k}\right) \text {-DNE }} \neg \neg \varphi_{1}^{\prime \prime} \wedge \neg \neg \varphi_{2}^{\prime \prime} \leftrightarrow \neg \neg\left(\varphi_{1}^{\prime \prime} \wedge \varphi_{2}^{\prime \prime}\right) \leftrightarrow \neg \neg \varphi^{\prime \prime}\right.$

The case of $\varphi_{1} \rightarrow \varphi_{2}$ : For the second item, assume $\varphi_{1} \rightarrow \varphi_{2} \in \mathrm{U}_{k}^{+}$. By Lemma 4.5, we have $\varphi_{1} \in \mathrm{E}_{k}^{+}$and $\varphi_{2} \in \mathrm{U}_{k}^{+}$. By induction hypothesis, there exist $\rho_{1}\left(x_{1}\right), \rho_{2}\left(x_{2}\right) \in \Sigma_{k-1}$ such that HA $+\neg \neg\left(\Pi_{k} \vee \Pi_{k}\right)$-DNE $\vdash \neg \varphi_{1} \leftrightarrow$ $\neg \neg \forall x_{1} \rho_{1}\left(x_{1}\right)$ and $\mathrm{HA}+\left(\Pi_{k} \vee \Pi_{k}\right)$-DNE $\vdash \varphi_{2} \leftrightarrow \forall x_{2} \rho_{2}\left(x_{2}\right)$. By Lemma 4.5, we have that $\neg \rho_{1}\left(x_{1}\right) \rightarrow \rho_{2}\left(x_{2}\right)$ is in $\mathrm{E}_{k-1}^{+}$. Then, by the item 1 for $k-1$, there exists $\xi\left(x_{1}, x_{2}\right) \in \Sigma_{k-1}$ such that

$$
\mathrm{HA}+\Sigma_{k-1}-\mathrm{DNE}+\mathrm{U}_{k-1}^{+}-\mathrm{DNS} \vdash \xi\left(x_{1}, x_{2}\right) \leftrightarrow\left(\neg \rho_{1}\left(x_{1}\right) \rightarrow \rho_{2}\left(x_{2}\right)\right) .
$$


Then, using Lemma 5.2 and (2), we have that $\mathrm{HA}+\left(\Pi_{k} \vee \Pi_{k}\right)$-DNE proves

$$
\begin{aligned}
& \varphi_{1} \rightarrow \varphi_{2} \\
& \text { [I.H.] }\left({\overleftrightarrow{\Pi_{k} \vee \Pi_{k}}}_{\text {-DNE }} \quad \varphi_{1} \rightarrow \forall x_{2} \rho_{2}\left(x_{2}\right)\right. \\
& \underset{\Pi_{k} \text {-DNE }}{\stackrel{\leftrightarrow}{\rightleftarrows}} \quad \varphi_{1} \rightarrow \neg \neg \forall x_{2} \rho_{2}\left(x_{2}\right) \\
& \begin{array}{rlrl} 
& \longleftrightarrow & & \neg \neg \varphi_{1} \rightarrow \neg \neg \forall x_{2} \rho_{2}\left(x_{2}\right) \\
\text { [I.H.] } \neg \neg\left(\overleftrightarrow{\left.\Pi_{k} \vee \Pi_{k}\right) \text {-DNE }}\right. & \neg \forall x_{1} \rho_{1}\left(x_{1}\right) \rightarrow \neg \neg \forall x_{2} \rho_{2}\left(x_{2}\right)
\end{array}
\end{aligned}
$$

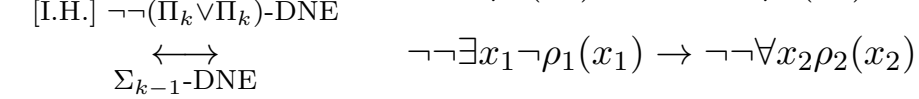

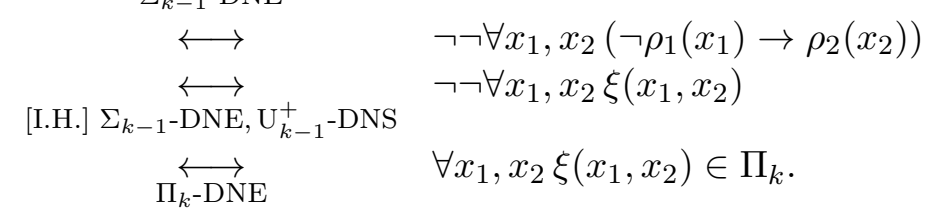

For the first and third items, assume $\varphi_{1} \rightarrow \varphi_{2} \in \mathrm{E}_{k}^{+}$. By Lemma 4.5, we have $\varphi_{1} \in \mathrm{U}_{k}^{+}$and $\varphi_{2} \in \mathrm{E}_{k}^{+}$. By induction hypothesis, there exists $\rho_{2}\left(x_{2}\right) \in$ $\Pi_{k-1}$ such that $\mathrm{HA}+\Sigma_{k}$-DNE $+\mathrm{U}_{k}^{+}$-DNS $\vdash \varphi_{2} \leftrightarrow \exists x_{2} \rho_{2}\left(x_{2}\right)$. In addition, by Lemma 5.1, there exists $\rho_{1}\left(x_{1}\right) \in \Sigma_{k-1}$ such that $\mathrm{HA}+\mathrm{U}_{k}^{+}$-DNS $\vdash \neg \neg \varphi_{1} \leftrightarrow$ $\neg \neg \forall x_{1} \rho_{1}\left(x_{1}\right)$. By Lemma4.5, we have $\neg \rho_{2}\left(x_{2}\right) \rightarrow \neg \rho_{1}\left(x_{1}\right)$ is in $\mathrm{U}_{k-1}^{+}$. Then, by the item 2 for $k-1$, there exists $\xi\left(x_{1}, x_{2}\right) \in \Pi_{k-1}$ such that

$$
\mathrm{HA}+\left(\Pi_{k-1} \vee \Pi_{k-1}\right)-\mathrm{DNE} \vdash \xi\left(x_{1}, x_{2}\right) \leftrightarrow\left(\neg \rho_{2}\left(x_{2}\right) \rightarrow \neg \rho_{1}\left(x_{1}\right)\right) .
$$

Then, using Lemma 5.2, we have that $\mathrm{HA}+\Sigma_{k}$-DNE $+\mathrm{U}_{k}^{+}$-DNS proves

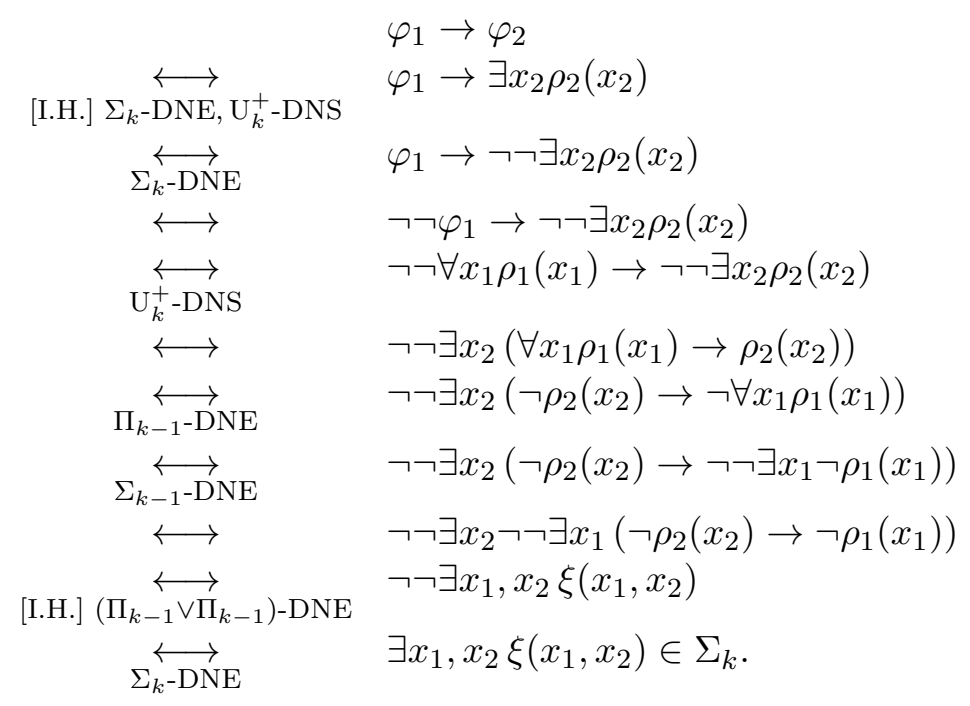

Thus we are done for the first item. For the third item, by induction hypothesis, there exist $\varphi_{1}^{\prime}, \varphi_{2}^{\prime} \in \Pi_{k}$ such that $\mathrm{HA}+\left(\Pi_{k} \vee \Pi_{k}\right)$-DNE $\vdash \varphi_{1} \leftrightarrow \varphi_{1}^{\prime}$ and HA $+\neg \neg\left(\Pi_{k} \vee \Pi_{k}\right)$-DNE $\vdash \neg \varphi_{2} \leftrightarrow \neg \neg \varphi_{2}^{\prime}$. On the other hand, by 
Lemma 4.3, there exists $\xi^{\prime} \in \Pi_{k}$ such that HA $\vdash \varphi_{1}^{\prime} \wedge \varphi_{2}^{\prime} \leftrightarrow \xi^{\prime}$. Then $\mathrm{HA}+\neg \neg\left(\Pi_{k} \vee \Pi_{k}\right)$-DNE proves

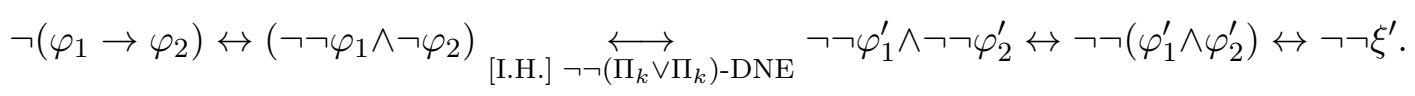

The case of $\forall x \varphi_{1}(x)$ : For the second item, assume $\forall x \varphi_{1}(x) \in \mathrm{U}_{k}^{+}$. By Lemma 4.5, we have $\varphi_{1}(x) \in \mathrm{U}_{k}^{+}$. By induction hypothesis, there exists $\varphi_{1}^{\prime}(x) \in \Pi_{k}$ such that $\mathrm{HA}+\left(\Pi_{k} \vee \Pi_{k}\right)$-DNE $\vdash \varphi_{1}(x) \leftrightarrow \varphi_{1}^{\prime}(x)$. Then $\forall x \varphi_{1}(x)$ is equivalent to $\forall x \varphi_{1}^{\prime}(x) \in \Pi_{k}$ over $\mathrm{HA}+\left(\Pi_{k} \vee \Pi_{k}\right)$-DNE.

For the first and third items, assume $\forall x \varphi_{1}(x) \in \mathrm{E}_{k}^{+}$. By Lemma 4.5, we have $\forall x \varphi_{1}(x) \in \mathrm{U}_{k-1}^{+}$. Then, by the item 2 for $k-1$, there exists $\xi \in \Pi_{k-1} \subseteq \Sigma_{k}$ (see Remark 2.5) such that

$$
\mathrm{HA}+\left(\Pi_{k-1} \vee \Pi_{k-1}\right)-\mathrm{DNE} \vdash \forall x \varphi_{1}(x) \leftrightarrow \xi .
$$

By Lemma 5.2, we are done for the first item. For the third item, by Lemma 4.7, there exists $\xi^{\prime} \in \Sigma_{k-1} \subseteq \Pi_{k}$ (see Remark 2.5) such that HA + $\Sigma_{k-1}$-DNE $\vdash \neg \xi \leftrightarrow \xi^{\prime}$. By Corollary 4.2, we have HA $+\neg \neg \Sigma_{k-1}$-DNE $\vdash$ $\neg \xi \leftrightarrow \neg \neg \xi^{\prime}$. In addition,

$$
\mathrm{HA}+\neg \neg\left(\Pi_{k-1} \vee \Pi_{k-1}\right) \text {-DNE } \vdash \neg \neg \forall x \varphi_{1}(x) \leftrightarrow \neg \neg \xi
$$

follows from (3). Then, by Lemma 5.2 , we have that $\mathrm{HA}+\neg \neg\left(\Pi_{k} \vee \Pi_{k}\right)$-DNE proves

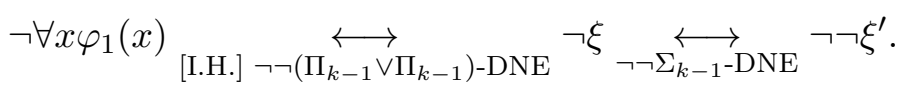

Thus we have shown the third item.

The case of $\exists x \varphi_{1}(x)$ : For the second item, assume $\exists x \varphi_{1}(x) \in \mathrm{U}_{k}^{+}$. By Lemma 4.5, we have $\exists x \varphi_{1}(x) \in \mathrm{E}_{k-1}^{+}$. Then, by the item 1 for $k-1$, there exists $\xi \in \Sigma_{k-1} \subseteq \Pi_{k}$ (see Remark 2.5) such that HA $+\Sigma_{k-1}$-DNE + $\mathrm{U}_{k-1}^{+}$-DNS $\vdash \exists x \varphi_{1}(x) \leftrightarrow \xi$. By Lemma 5.2 and (2), we have HA $+\left(\Pi_{k} \vee \Pi_{k}\right)$-DNE $\vdash$ $\exists x \varphi_{1}(x) \leftrightarrow \xi$.

For the first and third items, assume $\exists x \varphi_{1}(x) \in \mathrm{E}_{k}^{+}$. By Lemma 4.5, we have $\varphi_{1}(x) \in \mathrm{E}_{k}^{+}$. By induction hypothesis, there exist $\varphi_{1}^{\prime}(x) \in \Sigma_{k}$ and $\varphi_{1}^{\prime \prime}(x) \in \Pi_{k}$ such that $\mathrm{HA}+\Sigma_{k}$-DNE $+\mathrm{U}_{k}^{+}$-DNS $\vdash \varphi_{1}(x) \leftrightarrow \varphi_{1}^{\prime}(x)$ and $\mathrm{HA}+\neg \neg\left(\Pi_{k} \vee \Pi_{k}\right)$-DNE $\vdash \neg \varphi_{1}(x) \leftrightarrow \neg \neg \varphi_{1}^{\prime \prime}(x)$. Then $\exists x \varphi_{1}(x)$ is equivalent to $\exists x \varphi_{1}^{\prime}(x) \in \Sigma_{k}$ over $\mathrm{HA}+\Sigma_{k}$-DNE $+\mathrm{U}_{k}^{+}$-DNS. Thus we are done for the first item. For the third item, since $\mathrm{HA}+\neg \neg\left(\Pi_{k} \vee \Pi_{k}\right)$-DNE proves

$$
\neg \exists x \varphi_{1}(x) \leftrightarrow \forall x \neg \varphi_{1}(x){ }_{[\mathrm{I} . \mathrm{H} .]} \underset{\neg\left(\Pi_{k} \vee \Pi_{k}\right) \text {-DNE }}{\longrightarrow} \forall x \neg \neg \varphi_{1}^{\prime \prime}(x),
$$

we have that $\mathrm{HA}+\neg \neg\left(\Pi_{k} \vee \Pi_{k}\right)$-DNE proves

$$
\neg \exists x \varphi_{1}(x) \leftrightarrow \neg \neg \forall x \neg \neg \varphi_{1}^{\prime \prime}(x) .
$$

On the other hand, the latter is equivalent to $\neg \neg \forall x \varphi_{1}^{\prime \prime}(x)$ in the presence of $\neg \neg \Pi_{k}$-DNE. Thus we have HA $+\neg \neg\left(\Pi_{k} \vee \Pi_{k}\right)$-DNE $\vdash \neg \exists x \varphi_{1}(x) \leftrightarrow$ $\neg \neg \forall x \varphi_{1}^{\prime \prime}(x)$. 
Corollary 5.4. HA $+\neg \neg\left(\Pi_{k} \vee \Pi_{k}\right)$-DNE $\vdash \mathrm{U}_{k}^{+}$-DNS.

Proof. Since $\mathrm{U}_{k}^{+}$-DNS is intuitionistically equivalent to $\neg \neg \mathrm{U}_{k}^{+}$-DNS (see Remark 2.8), it suffices to show $\mathrm{HA}+\left(\Pi_{k} \vee \Pi_{k}\right)$-DNE $\vdash \mathrm{U}_{k}^{+}$-DNS. By Theorem 5.3. (22), any formulas $\varphi \in \mathrm{U}_{k}^{+}$is equivalent to some $\varphi^{\prime} \in \Pi_{k}$ such that $\mathrm{FV}(\varphi)=\mathrm{FV}\left(\varphi^{\prime}\right)$ over HA $+\left(\Pi_{k} \vee \Pi_{k}\right)$-DNE. Since HA $+\Pi_{k}$-DNE $\vdash$ $\Pi_{k}$-DNS, we have $\mathrm{HA}+\left(\Pi_{k} \vee \Pi_{k}\right)$-DNE $\vdash \mathrm{U}_{k}^{+}$-DNS.

Remark 5.5. Corollary 5.4 shows that Lemma 5.1 (equivalent to item 1 in the proof of Lemma 5.1) is a stronger statement of the item 3 in the proof of Theorem 5.3. On the other hand, it is still open whether $\mathrm{HA}+\mathrm{U}_{k}^{+}$-DNS is a proper subsystem of $\mathrm{HA}+\neg \neg\left(\Pi_{k} \vee \Pi_{k}\right)$-DNE.

Remark 5.6. It follows from Theorem 5.3 and the results in Section 3 that $\mathrm{HA}+\Sigma_{1}$-DNE does not prove $\mathrm{U}_{1}^{+}$-DNS.

At the end of this section, we study the prenex normal form theorem for formulas which do not contain the disjunction $V$. In fact, the proof of Theorem 5.3 suggests that the unusual form $\left(\Pi_{k} \vee \Pi_{k}\right)$-DNE of the double negation elimination is caused from the argument especially in the case of $\varphi_{1} \vee \varphi_{2}$. On the other hand, if a formula $\varphi$ does not contain $\vee$, one can intuitionistically derive the original formula $\varphi$ from a formula of the prenex normal form which is classically equivalent to $\varphi$ (cf. [9, Lemma 6.2.1]). Then the proof of the prenex normal form theorem for those formulas becomes to be fairly simple.

Theorem 5.7. For each natural number $k$ and a formula $\varphi$ (possibly containing free variables) which does not contain $\vee$, the following hold:

1. if $\varphi \in \mathrm{E}_{k}^{+}$, then there exists $\varphi^{\prime} \in \Sigma_{k}$ such that $\mathrm{FV}(\varphi)=\mathrm{FV}\left(\varphi^{\prime}\right)$ and

$$
\mathrm{HA}+\Sigma_{k}-\mathrm{DNE} \vdash \varphi \leftrightarrow \varphi^{\prime} ;
$$

2. if $\varphi \in \mathrm{U}_{k}^{+}$, then there exists $\varphi^{\prime} \in \Pi_{k}$ such that $\mathrm{FV}(\varphi)=\mathrm{FV}\left(\varphi^{\prime}\right)$ and

$$
\left.\mathrm{HA}+\Sigma_{k-1} \text {-DNE (HA if } k=0\right) \vdash \varphi \leftrightarrow \varphi^{\prime} .
$$

Proof. We mimic the proof of Theorem 5.3. Thus we first prepare the following auxiliary assertion (which is in fact a consequence from the item 2):

3. if $\varphi \in \mathrm{E}_{k}^{+}$, then there exists $\varphi^{\prime} \in \Pi_{k}$ such that $\mathrm{FV}(\varphi)=\mathrm{FV}\left(\varphi^{\prime}\right)$ and

$$
\mathrm{HA}+\neg \neg \Sigma_{k-1}-\mathrm{DNE} \vdash \neg \varphi \leftrightarrow \neg \neg \varphi^{\prime} .
$$

Then we show the items 1, 2 and 3 by induction on $k$ simultaneously. The base case is trivial. Most of the parts for the induction step is the same as those for Theorem 5.3. The same proof works since the logical principle in 
the items 1 and 2 implies both of them for $k-1$ and the logical principle in the item 3 is the double negation of the logical principle in the item 2 as in Theorem 5.3 .

Only the difference with the proof of Theorem 5.3 is in proving the item 1 for $\varphi: \equiv \varphi_{1} \rightarrow \varphi_{2} \in \mathrm{E}_{k}^{+}$, where we use Lemma 5.1. Here one can use the item 2 instead of Lemma 5.1. This is because $\Sigma_{k}$ DNE includes $\Sigma_{k-1}$-DNE while the verification theory of the item 1 in Theorem 5.3 contains the verification theory of Lemma [5.1. To be absolutely clear, we present the proof of this part: Let $\varphi_{1} \rightarrow \varphi_{2} \in \mathrm{E}_{k}^{+}$. By Lemma 4.5, we have $\varphi_{1} \in \mathrm{U}_{k}^{+}$and $\varphi_{2} \in \mathrm{E}_{k}^{+}$. By induction hypothesis, there exists $\rho_{1}\left(x_{1}\right) \in \Sigma_{k-1}$ and $\rho_{2}\left(x_{2}\right) \in \Pi_{k-1}$ such that HA $+\Sigma_{k-1}$-DNE $\vdash \varphi_{1} \leftrightarrow \forall x_{1} \rho_{1}\left(x_{1}\right)$ and HA $+\Sigma_{k}$-DNE $\vdash \varphi_{2} \leftrightarrow$ $\exists x_{2} \rho_{2}\left(x_{2}\right)$. By Lemma 4.5, we have $\neg \rho_{2}\left(x_{2}\right) \rightarrow \neg \rho_{1}\left(x_{1}\right)$ is in $\mathrm{U}_{k-1}^{+}$. Then, by the item 2 for $k-1$, there exists $\xi\left(x_{1}, x_{2}\right) \in \Pi_{k-1}$ such that

$$
\mathrm{HA}+\Sigma_{k-2} \text {-DNE } \vdash \xi\left(x_{1}, x_{2}\right) \leftrightarrow\left(\neg \rho_{2}\left(x_{2}\right) \rightarrow \neg \rho_{1}\left(x_{1}\right)\right) .
$$

Then $\mathrm{HA}+\Sigma_{k}$-DNE proves

$$
\begin{aligned}
& \begin{aligned}
& \varphi_{1} \rightarrow \varphi_{2} \\
& \text { [I.H.] } \overleftrightarrow{\Sigma_{k} \text {-DNE }} \quad \varphi_{1} \rightarrow \exists x_{2} \rho_{2}\left(x_{2}\right)
\end{aligned}
\end{aligned}
$$

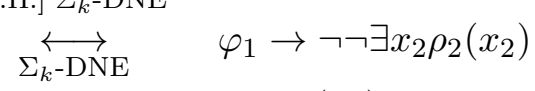

$$
\begin{aligned}
& \text { [I.H.] } \stackrel{\Sigma_{k-1} \text {-DNE }}{\longrightarrow} \forall x_{1} \rho_{1}\left(x_{1}\right) \rightarrow \neg \neg \exists x_{2} \rho_{2}\left(x_{2}\right) \\
& \longleftrightarrow \quad \neg \neg \exists x_{2}\left(\forall x_{1} \rho_{1}\left(x_{1}\right) \rightarrow \rho_{2}\left(x_{2}\right)\right) \\
& \underset{\Pi_{k-1} \text {-DNE }}{\longleftrightarrow} \neg \neg \exists x_{2}\left(\neg \rho_{2}\left(x_{2}\right) \rightarrow \neg \forall x_{1} \rho_{1}\left(x_{1}\right)\right) \\
& \underset{\Sigma_{k-1} \text {-DNE }}{\longleftrightarrow} \neg \neg \exists x_{2}\left(\neg \rho_{2}\left(x_{2}\right) \rightarrow \neg \neg \exists x_{1} \neg \rho_{1}\left(x_{1}\right)\right) \\
& \stackrel{\longleftrightarrow}{\longleftrightarrow} \neg \neg \exists x_{2} \neg \neg \exists x_{1}\left(\neg \rho_{2}\left(x_{2}\right) \rightarrow \neg \rho_{1}\left(x_{1}\right)\right) \\
& \text { [I.H.] } \overleftrightarrow{\Sigma_{k-2} \text {-DNE }} \neg \neg \exists x_{1}, x_{2} \xi\left(x_{1}, x_{2}\right) \\
& \underset{\Sigma_{k} \text {-DNE }}{\overleftrightarrow{\longrightarrow}} \exists x_{1}, x_{2} \xi\left(x_{1}, x_{2}\right) \in \Sigma_{k}
\end{aligned}
$$

Remark 5.8. It follows from Theorem 5.3 and Corollary 5.4 that $\mathrm{E}_{k}^{+}$-LEM, $\mathrm{U}_{k}^{+}$-LEM and $\mathrm{U}_{k}^{+}$-DNE are equivalent to $\Sigma_{k}$-LEM, $\Pi_{k}$-LEM and $\left(\Pi_{k} \vee \Pi_{k}\right)$-DNE respectively over HA (cf. [1, Corollary 2.9]). This may not be the case for $\mathrm{E}_{k}^{+}$-DNE and $\Sigma_{k}$-DNE. On the other hand, Theorem 5.7 implies that $\mathrm{HA}+\Sigma_{k}$-DNE proves the double negation elimination for all formulas in $\mathrm{E}_{k}^{+}$ which do not contain $\vee$.

\section{A conservation result}

In this section, we generalize a well-known fact that $\mathrm{PA}$ is $\Pi_{2}$-conservative over HA in the context of semi-classical arithmetic (see Theorem 6.14). The 
fact is normally shown by applying the negative translation followed by the Friedman A-translation (see e.g. [5, Chapter 14]). As for the negative translation, there are several equivalent forms (see [7, Section 1.10.1]). Here we employ Kuroda's negative translation among them.

Definition 6.1 (cf. Definition 10.1 in [5]). Let $\varphi$ be a HA-formula. Then its negative translation $\varphi^{N}$ is defined as $\varphi^{N}: \equiv \neg \neg \varphi_{N}$, where $\varphi_{N}$ is defined by induction on the logical structure of $\varphi$ as follows:

- $\left(\varphi_{\mathrm{p}}\right)_{N}: \equiv \varphi_{\mathrm{p}}$ if $\varphi_{\mathrm{p}}$ is a prime formula;

- $\left(\varphi_{1} \circ \varphi_{2}\right)_{N}: \equiv\left(\varphi_{1}\right)_{N} \circ\left(\varphi_{2}\right)_{N}$, where $\circ \in\{\wedge, \vee, \rightarrow\}$;

- $\left(\exists x \varphi_{1}\right)_{N}: \equiv \exists x\left(\varphi_{1}\right)_{N}$;

- $\left(\forall x \varphi_{1}\right)_{N}: \equiv \forall x \neg \neg\left(\varphi_{1}\right)_{N}$.

Remark 6.2. By induction on the structure of formulas, one can show FV $(\varphi)=$ $\mathrm{FV}\left(\varphi_{N}\right)=\mathrm{FV}\left(\varphi^{N}\right)$ for all formulas $\varphi$. When it is clear from the context, we suppress the argument on free variables.

Lemma 6.3. For any HA-formula $\varphi$ of the prenex normal form, HA proves $\varphi \rightarrow \varphi_{N}$.

Proof. By induction on the structure of formulas of the prenex normal form.

Proposition 6.4. For any HA-formula $\varphi$, if $\mathrm{PA} \vdash \varphi$, then $\mathrm{HA} \vdash \varphi^{N}$.

Proof. By induction on the length of the derivations (see the proof of [5, Proposition 10.3]).

Lemma 6.5. Let $k$ be a natural number.

1. For any HA-formula $\varphi \in \Sigma_{k}$, HA $+\Sigma_{k}$-DNE proves $\varphi^{N} \leftrightarrow \varphi$.

2. For any $\mathrm{HA}$-formula $\varphi \in \Pi_{k}, \mathrm{HA}+\Sigma_{k-1}$-DNE (HA if $k=0$ ) proves $\varphi^{N} \leftrightarrow \varphi$.

Proof. By simultaneous induction on $k$. The base case is trivial. For the induction step, assume the items 1 and 2 for $k$ to show those for $k+1$. For the first item, let $\exists x \varphi_{1} \in \Sigma_{k+1}$ where $\varphi_{1} \in \Pi_{k}$. We have that HA $+\Sigma_{k+1}$-DNE proves

$\left(\exists x \varphi_{1}\right)^{N} \equiv \neg \neg \exists x\left(\varphi_{1}\right)_{N} \leftrightarrow \neg \neg \exists x \neg \neg\left(\varphi_{1}\right)_{N}{ }_{[\text {I.H.] }] \Sigma_{k-1} \text {-DNE }}^{\overleftrightarrow{\longleftrightarrow}} \neg \neg \exists x \varphi_{1} \underset{\Sigma_{k+1} \text {-DNE }}{\overleftrightarrow{\longleftrightarrow}} \exists x \varphi_{1}$

For the second item, let $\forall x \varphi_{1} \in \Pi_{k+1}$ where $\varphi_{1} \in \Sigma_{k}$. Since $\Pi_{k+1}$-DNE is derived from $\Sigma_{k}$-DNE (see Lemma 5.2.(4)), we have that $\mathrm{HA}+\Sigma_{k}$-DNE proves

$$
\left(\forall x \varphi_{1}\right)^{N} \equiv \neg \neg \forall x \neg \neg\left(\varphi_{1}\right)_{N} \underset{[\text { I.H.] }] \Sigma_{k} \text {-DNE }}{\longleftrightarrow} \neg \neg \forall x \varphi_{1} \underset{\Pi_{k+1} \text {-DNE }}{\overleftrightarrow{\longrightarrow}} \forall x \varphi_{1}
$$


Let $\mathrm{HA}^{*}$ denote $\mathrm{HA}$ in the extended language where a predicate symbol * of arity 0 , which behaves as a place holder, is added. In particular, $\mathrm{HA}^{*}$ has $\perp \rightarrow *$ as an axiom. To make our arguments absolutely clear, we prefer to add the distinguished new predicate $*$ rather than discussing about Atranslation inside the original language as done in [2,5].

Definition 6.6 (A-translation [2]). For a HA-formula $\varphi$, we define $\varphi^{*}$ as a formula obtained from $\varphi$ by replacing all the prime formulas $\varphi_{\mathrm{p}}$ in $\varphi$ with $\varphi_{\mathrm{p}} \vee *$. Officially, $\varphi^{*}$ is defined by induction on the logical structure of $\varphi$ as in Definition 6.1. In particular, $\perp^{*}: \equiv(\perp \vee *)$, which is equivalent to $*$ over HA* $^{*}$. In what follows, $\neg_{*} \varphi$ denotes $\varphi \rightarrow *$.

Remark 6.7. By induction on the structure of formulas, one can show FV $(\varphi)=$ $\mathrm{FV}\left(\varphi^{*}\right)$ for all HA-formulas $\varphi$.

Proposition 6.8 (Cf. Lemma 2 in [2]). For any $\mathrm{HA}$-formula $\varphi$, if $\mathrm{HA} \vdash \varphi$, then $\mathrm{HA}^{*} \vdash \varphi^{*}$.

Proof. By induction on the length of the derivations.

Remark 6.9. An analogous assertion of Proposition 6.8 holds for HA + $\Sigma_{1}$-LEM and $\mathrm{HA}^{*}+\Sigma_{1}$-LEM instead of $\mathrm{HA}$ and $\mathrm{HA}^{*}$ respectively (see [6, Lemma 3.1]).

The following substitution result is important in the application of the A-translation:

Lemma 6.10 (Cf. Theorem 6.2.4 in [9]). Let $X$ be a set of HA-sentences and $\varphi$ be a $\mathrm{HA}^{*}$-formula. If $\mathrm{HA}^{*}+X \vdash \varphi$, then $\mathrm{HA}+X \vdash \varphi[\psi / *]$ for any $\mathrm{HA}$-formula $\psi$ such that the free variables of $\psi$ are not bounded in $\varphi$, where $\varphi[\psi / *]$ is the HA-formula obtained from $\varphi$ by replacing all the occurrences of $*$ in $\varphi$ with $\psi$.

Proof. Fix a set $X$ of $\mathrm{HA}$-sentences. By induction on $k$, one can show straightforwardly that for any $k$ and any $\mathrm{HA}^{*}$-formula $\varphi$, if $\mathrm{HA}^{*}+X \vdash \varphi$ with the proof of length $k$, then $\mathrm{HA}+X \vdash \varphi[\psi / *]$ for any HA-formula $\psi$ such that the free variables of $\psi$ is not bounded in $\varphi$. The variable condition is used to verify the case of axioms and rules for quantifiers.

The following lemma is a key for our generalized conservation results.

Lemma 6.11. Let $k$ be a natural number.

1. For any $\mathrm{HA}$-formula $\varphi \in \Sigma_{k}, \mathrm{HA}^{*}+\Sigma_{k-1}$-LEM $\left(\mathrm{HA}^{*}\right.$ if $\left.k=0\right)$ proves $\left(\varphi_{N}\right)^{*} \leftrightarrow \varphi_{N} \vee *$.

2. For any HA-formula $\varphi \in \Pi_{k}, \mathrm{HA}^{*}+\Sigma_{k}$-LEM proves $\left(\varphi_{N}\right)^{*} \leftrightarrow \varphi_{N} \vee *$. 
Proof. We show the items 1 and 2 simultaneously by induction on $k$.

The base case: Since every quantifier-free formula $\varphi_{\mathrm{qf}} \operatorname{such}$ that $\mathrm{FV}\left(\varphi_{\mathrm{qf}}\right)=$ $\{\bar{x}\}$ is equivalent to a prime formula $t(\bar{x})=0$ for some closed term $t$ (see e.g. [5, Proposition 3.8]), by Proposition 6.8, it suffices to show the assertions only for prime formulas. Since $\left(\left(\varphi_{\mathrm{p}}\right)_{N}\right)^{*} \equiv \varphi_{\mathrm{p}}{ }^{*} \equiv \varphi_{\mathrm{p}} \vee * \equiv\left(\varphi_{\mathrm{p}}\right)_{N} \vee *$, we are done.

The induction step: Assume that the items 1 and 2 hold for $k$. We first show the item 1 for $k+1$. Let $\varphi_{1} \in \Pi_{k}$. Since

$$
\left(\left(\exists x \varphi_{1}\right)_{N}\right)^{*} \equiv\left(\exists x\left(\varphi_{1}\right)_{N}\right)^{*} \equiv \exists x\left(\left(\varphi_{1}\right)_{N}\right)^{*},
$$

by induction hypothesis, we have

$$
\mathrm{HA}^{*}+\Sigma_{k} \text { LEM } \vdash\left(\left(\exists x \varphi_{1}\right)_{N}\right)^{*} \leftrightarrow \exists x\left(\left(\varphi_{1}\right)_{N} \vee *\right) .
$$

Since HA* proves $\exists x\left(\left(\varphi_{1}\right)_{N} \vee *\right) \leftrightarrow\left(\exists x\left(\varphi_{1}\right)_{N} \vee *\right) \equiv\left(\left(\exists x \varphi_{1}\right)_{N} \vee *\right)$, we have

$$
\mathrm{HA}^{*}+\Sigma_{k} \text {-LEM } \vdash\left(\left(\exists x \varphi_{1}\right)_{N}\right)^{*} \leftrightarrow\left(\left(\exists x \varphi_{1}\right)_{N} \vee *\right) .
$$

Thus we have shown the item 1 for $k+1$.

Next, we show the item 2 for $k+1$. Let $\varphi_{2} \in \Sigma_{k}$. We shall show that $\mathrm{HA}^{*}+\Sigma_{k}$-DNE (and hence, $\mathrm{HA}^{*}+\Sigma_{k+1}$-LEM) proves $\left(\forall x \varphi_{2}\right)_{N} \mathrm{~V} * \rightarrow$ $\left(\left(\forall x \varphi_{2}\right)_{N}\right)^{*}$. By Lemma 6.5), (11), we have

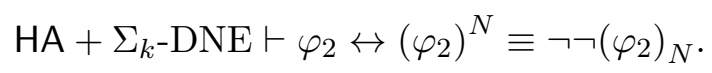

Then we have that $\mathrm{HA}^{*}+\Sigma_{k}$-DNE proves

$$
\left(\forall x \varphi_{2}\right)_{N} \vee * \equiv\left(\forall x \neg \neg\left(\varphi_{2}\right)_{N} \vee *\right) \leftrightarrow \forall x \varphi_{2} \vee * .
$$

By Lemma 6.3. HA proves $\varphi_{2} \rightarrow\left(\varphi_{2}\right)_{N}$. Then, using induction hypothesis and the fact that $\Sigma_{k}$-DNE derives $\Sigma_{k-1}$-LEM, we have that $\mathrm{HA}^{*}+\Sigma_{k}$-DNE proves

$$
\begin{array}{rll}
\left(\forall x \varphi_{2}\right)_{N} \vee * & \overleftrightarrow{\Sigma_{k} \text {-DNE }} & \forall x \varphi_{2} \vee * \\
\longrightarrow & \forall x\left(\varphi_{2}\right)_{N} \vee * \\
\text { [I.H.] } & \overleftrightarrow{\Sigma_{k-1} \text {-LEM }} & \forall x\left(\left(\varphi_{2}\right)_{N} \vee *\right) \\
& \longleftrightarrow & \forall x\left(\left(\left(\varphi_{2}\right)_{N}\right)^{*}\right. \\
& \left.\left.\left(\left(\varphi_{2}\right)_{N}\right)^{*} \rightarrow *\right) \rightarrow *\right) \\
& & \left.\left(\forall \varphi_{2}\right)_{N}\right)^{*} .
\end{array}
$$

In the following, we show the converse direction:

$$
\mathrm{HA}^{*}+\Sigma_{k+1}-\mathrm{LEM} \vdash\left(\left(\forall x \varphi_{2}\right)_{N}\right)^{*} \rightarrow\left(\forall x \varphi_{2}\right)_{N} \vee * .
$$

Reason in $\mathrm{HA}^{*}+\Sigma_{k+1}$-LEM. Suppose $\left(\left(\forall x \varphi_{2}\right)_{N}\right)^{*}$, equivalently,

$$
\forall x\left(\left(\left(\left(\varphi_{2}\right)_{N}\right)^{*} \rightarrow *\right) \rightarrow *\right) .
$$


By induction hypothesis, (6) is equivalent to $\forall x\left(\left(\left(\varphi_{2}\right)_{N} \vee * \rightarrow *\right) \rightarrow *\right)$, which is intuitionistically equivalent to

$$
\forall x\left(\left(\left(\varphi_{2}\right)_{N} \rightarrow *\right) \rightarrow *\right) .
$$

Then we have

$$
\exists x \neg\left(\varphi_{2}\right)_{N} \rightarrow * .
$$

By Lemma 4.7.(2), there exists $\psi_{2} \in \Pi_{k}$ such that $\mathrm{FV}\left(\varphi_{2}\right)=\mathrm{FV}\left(\psi_{2}\right)$ and $\neg \varphi_{2}$ is equivalent to $\psi_{2}$. Since $\exists x \psi_{2} \in \Sigma_{k+1}$, by $\Sigma_{k+1}$-LEM, we have $\exists x \psi_{2} \vee \neg \exists x \psi_{2}$, and hence,

$$
\exists x \neg \varphi_{2} \vee \forall x \neg \neg \varphi_{2} .
$$

Then, by (4), we obtain

$$
\exists x \neg\left(\varphi_{2}\right)_{N} \vee \forall x \neg \neg\left(\varphi_{2}\right)_{N} .
$$

In the former case, we have $*$ by (77). In the latter case, we have $\left(\forall x \varphi_{2}\right)_{N}$. Thus we have shown (5).

Lemma 6.12. Let $\varphi$ be a $\mathrm{HA}^{*}$-formula.

1. $\mathrm{HA}^{*} \vdash \varphi \rightarrow \neg_{*} \neg_{*} \varphi$.

2. $\mathrm{HA}^{*} \vdash \forall x \neg_{*} \varphi \leftrightarrow \neg_{*} \exists x \varphi$.

3. $\mathrm{HA}^{*} \vdash \neg_{*} \neg_{*} \neg_{*} \varphi \rightarrow \neg_{*} \varphi$.

4. $\mathrm{HA}^{*} \vdash \exists x \neg_{*} \neg_{*} \varphi \rightarrow \neg_{*} \neg_{*} \exists x \varphi$.

Proof. (11), (2) and (3) are immediate from the definition of $\neg_{*}$ (see Definition (6.6). (4) follows from (11), (21) and (3).

Lemma 6.13. For any HA-formula $\varphi$ of the prenex normal form, $\mathrm{HA}^{*} \vdash$ $\varphi \rightarrow\left(\varphi^{N}\right)^{*}$.

Proof. Since there exists a closed term $t$ such that HA $\vdash \varphi_{\mathrm{qf}}\left(x_{1}, \ldots, x_{k}\right) \leftrightarrow$ $t\left(x_{1}, \ldots, x_{k}\right)=0$ for each quantifier-free formula $\varphi_{\mathrm{qf}}$ such that $\mathrm{FV}\left(\varphi_{\mathrm{qf}}\right)=$ $\left\{x_{1}, \ldots, x_{k}\right\}$ (see e.g. [5, Proposition 3.8]), by Proposition 6.4 and Proposition 6.8, one can assume that formulas of the prenex normal form consist of the formulas of form $Q_{1} x_{1} \ldots Q_{k} x_{k} \varphi_{\mathrm{p}}$ where $Q_{i} \mathrm{~s}$ are quantifiers and $\varphi_{\mathrm{p}}$ is prime. We show our assertion by induction on the structure of formulas of this form.

For a prime formula $\varphi_{\mathrm{p}}$, it is trivial to see that $\mathrm{HA}^{*}$ proves

$$
\varphi_{\mathrm{p}} \rightarrow \varphi_{\mathrm{p}} \vee * \rightarrow \neg_{*} \neg_{*}\left(\varphi_{\mathrm{p}} \vee *\right) \leftrightarrow\left(\left(\varphi_{\mathrm{p}}\right)^{N}\right)^{*}
$$


Assume the assertion for $\varphi$. Then, using Lemma 6.12, HA* proves

$$
\begin{array}{r}
\exists x \varphi \underset{\text { [I.H.] }}{\longrightarrow} \exists x\left(\varphi^{N}\right)^{*} \equiv \exists x\left(\neg \neg \varphi_{N}\right)^{*} \leftrightarrow \exists x \neg_{*} \neg_{*}\left(\varphi_{N}\right)^{*} \rightarrow \neg_{*} \neg * \exists x\left(\varphi_{N}\right)^{*} \\
\leftrightarrow\left((\exists x \varphi)^{N}\right)^{*}
\end{array}
$$

and

$$
\begin{array}{r}
\left.\forall x \varphi \underset{\text { [I.H.] }}{\longrightarrow} \forall x\left(\varphi^{N}\right)^{*} \equiv \forall x\left(\neg \neg \varphi_{N}\right)^{*} \leftrightarrow \forall x \neg_{*}\right\urcorner_{*}\left(\varphi_{N}\right)^{*} \rightarrow \neg_{*} \neg * \forall x \neg_{*} \neg *\left(\varphi_{N}\right)^{*} \\
\leftrightarrow\left((\forall x \varphi)^{N}\right)^{*} .
\end{array}
$$

Theorem 6.14. Let $k$ be a natural number. For any formulas $\varphi \in \Pi_{k+2}$ and $\psi$ of the prenex normal form, if PA $\vdash \psi \rightarrow \varphi$, then $\mathrm{HA}+\Sigma_{k}$-LEM $\vdash \psi \rightarrow \varphi$.

Proof. Let $\varphi: \equiv \forall x \exists y \varphi_{1}$ where $\varphi_{1} \in \Pi_{k}$. Since one can freely replace the bound variables, assume that the free variables of $\exists y \varphi_{1}$ are not bounded in $\psi$ and $x$ does not occur in $\psi$ without loss of generality.

Suppose PA $\vdash \psi \rightarrow \forall x \exists y \varphi_{1}$. By Proposition [6.4, we have that HA proves $\neg \neg\left(\psi_{N} \rightarrow \forall x \neg \neg \exists y\left(\varphi_{1}\right)_{N}\right)$, which is intuitionistically equivalent to $\neg \neg \psi_{N} \rightarrow \forall x \neg \neg \exists y\left(\varphi_{1}\right)_{N}$, namely, $\psi^{N} \rightarrow \forall x \neg \neg \exists y\left(\varphi_{1}\right)_{N}$. Then we have

$$
\mathrm{HA} \vdash \psi^{N} \rightarrow \neg \neg \exists y\left(\varphi_{1}\right)_{N} .
$$

By Proposition 6.8, we have

$$
\mathrm{HA}^{*} \vdash\left(\psi^{N}\right)^{*} \rightarrow \neg * \neg * \exists y\left(\left(\varphi_{1}\right)_{N}\right)^{*},
$$

and hence,

$$
\mathrm{HA}^{*} \vdash \psi \rightarrow \neg * \neg * \exists y\left(\left(\varphi_{1}\right)_{N}\right)^{*}
$$

by Lemma 6.13. Then, by Lemma 6.11,(2), we have that $\mathrm{HA}^{*}+\Sigma_{k}$-LEM proves

$$
\psi \rightarrow \neg * \neg * \exists y\left(\left(\varphi_{1}\right)_{N} \vee *\right),
$$

which is intuitionistically equivalent to

$$
\psi \rightarrow \neg * \neg * \exists y\left(\varphi_{1}\right)_{N} .
$$

Since the free variables of $\exists y \varphi_{1}$ are not bounded in $\psi$, using Lemma 6.10 with Remark 6.2, we have

$$
\mathrm{HA}+\Sigma_{k} \text { LEM } \vdash \psi \rightarrow\left(\left(\exists y\left(\varphi_{1}\right)_{N} \rightarrow \exists y \varphi_{1}\right) \rightarrow \exists y \varphi_{1}\right) .
$$

On the other hand, by Lemma 6.5.(2) and the fact that $\Sigma_{k}$-LEM derives $\Sigma_{k}$-DNE, we have that HA $+\Sigma_{k}$-LEM proves

$$
\left(\varphi_{1}\right)_{N} \rightarrow \neg \neg\left(\varphi_{1}\right)_{N} \equiv\left(\varphi_{1}\right)^{N} \underset{\Sigma_{k-1-D N E}}{\longleftrightarrow} \varphi_{1}
$$

and hence, $\exists y\left(\varphi_{1}\right)_{N} \rightarrow \exists y \varphi_{1}$. Then, by (8), we have HA $+\Sigma_{k}$-LEM $\vdash \psi \rightarrow$ $\exists y \varphi_{1}$. By our assumption, $x$ does not occur in $\psi$, and hence, $\mathrm{HA}+\Sigma_{k}$-LEM $\vdash$ $\psi \rightarrow \forall x \exists y \varphi_{1}$ follows. 
We have shown Theorem 6.14 in order to prove the optimality of our prenex normal form theorems in Section 5 (see Section 7). On the other hand, the conservation result on semi-classical arithmetic itself is interesting. This will be studied comprehensively in [4.

\section{Characterizations}

Notation 2. Let $T$ be an extension of HA. Let $\Gamma$ and $\Gamma^{\prime}$ be classes of HAformulas. Then $\operatorname{PNFT}_{T}\left(\Gamma, \Gamma^{\prime}\right)$ denotes the following statement: for any $\varphi \in \Gamma$, there exists $\varphi^{\prime} \in \Gamma^{\prime}$ such that $\mathrm{FV}(\varphi)=\mathrm{FV}\left(\varphi^{\prime}\right)$ and $T \vdash \varphi \leftrightarrow \varphi^{\prime}$.

Under this notation, Theorem 5.3 asserts (modulo Remark 2.2) that for a semi-classical theory $T$ containing $\mathrm{HA}+\left(\Pi_{k} \vee \Pi_{k}\right)$-DNE, $\operatorname{PNFT}_{T}\left(\mathrm{U}_{k^{\prime}}, \Pi_{k^{\prime}}\right)$ holds for all $k^{\prime} \leq k$ as well as the analogous assertion for $\mathrm{E}_{k}$ and $\Sigma_{k}$. It is natural to ask whether the verification theories are optimal. In this section, among other things (see Table 1), we show that this is exactly the case:

1. For a theory $T$ in-between HA and PA, $T \vdash\left(\Pi_{k} \vee \Pi_{k}\right)$-DNE if and only if $\mathrm{PNFT}_{T}\left(\mathrm{U}_{k^{\prime}}, \Pi_{k^{\prime}}\right)$ for all $k^{\prime} \leq k$. (Theorem 7.3 )

2. For a theory $T$ in-between $\mathrm{HA}+\Pi_{k-1}$-LEM (HA if $k=0$ ) and PA, $T \vdash \Sigma_{k^{-}}$DNE $+\mathrm{U}_{k}^{+}$-DNS if and only if $\operatorname{PNFT}_{T}\left(\mathrm{E}_{k^{\prime}}, \Sigma_{k^{\prime}}\right)$ for all $k^{\prime} \leq k$. (Theorem 7.11)

Lemma 7.1. Let $T$ be a theory in-between $\mathrm{HA}+\Sigma_{k-2}$-LEM $(\mathrm{HA}$ if $k<2)$ and $\mathrm{PA}$. If $\mathrm{PNFT}_{T}\left(\mathrm{U}_{k}, \Pi_{k}\right)$, then $T \vdash\left(\Pi_{k} \vee \Pi_{k}\right)$-DNE.

Proof. Fix an instance of $\left(\Pi_{k} \vee \Pi_{k}\right)$-DNE

$$
\varphi: \equiv \forall x\left(\neg \neg\left(\varphi_{1}(x) \vee \varphi_{2}(x)\right) \rightarrow \varphi_{1}(x) \vee \varphi_{2}(x)\right),
$$

where $\varphi_{1}(x), \varphi_{2}(x) \in \Pi_{k}(x)$. Since $\neg \neg\left(\varphi_{1}(x) \vee \varphi_{2}(x)\right)$ and $\varphi_{1}(x) \vee \varphi_{2}(x)$ are in $\mathrm{U}_{k}$, by our assumption, there exist $\rho(x)$ and $\rho^{\prime}(x)$ in $\Pi_{k}(x)$ such that $T$ proves $\rho(x) \leftrightarrow \varphi_{1}(x) \vee \varphi_{2}(x)$ and $\rho^{\prime}(x) \leftrightarrow \neg \neg\left(\varphi_{1}(x) \vee \varphi_{2}(x)\right)$. Since PA $\vdash \varphi$ and PA is an extension of $T$, we have PA $\vdash \rho^{\prime}(x) \rightarrow \rho(x)$. By Theorem 6.14, we have that HA $+\Sigma_{k-2}$-LEM proves $\rho^{\prime}(x) \rightarrow \rho(x)$, and hence, $\forall x\left(\rho^{\prime}(x) \rightarrow \rho(x)\right)$. Since $T$ is an extension of HA $+\Sigma_{k-2}$-LEM, we have $T \vdash \forall x\left(\rho^{\prime}(x) \rightarrow \rho(x)\right)$, and hence, $T \vdash \varphi$.

Lemma 7.2. Let $T$ be a theory in-between $\mathrm{HA}$ and $\mathrm{PA}$. If $\mathrm{PNFT}_{T}\left(\mathrm{U}_{k^{\prime}}, \Pi_{k^{\prime}}\right)$ for all $k^{\prime} \leq k$, then $T \vdash \Sigma_{k-1}$-LEM.

Proof. By induction on $k$. The base case is trivial. For the induction step, assume $\operatorname{PNFT}_{T}\left(\mathrm{U}_{k^{\prime}}, \Pi_{k^{\prime}}\right)$ for all $k^{\prime} \leq k+1$. Then, by induction hypothesis, we have $T \vdash \Sigma_{k-1}$-LEM. Fix an instance of $\Sigma_{k}$-LEM

$$
\varphi: \equiv \forall x\left(\varphi_{1}(x) \vee \neg \varphi_{1}(x)\right)
$$


where $\varphi_{1}(x) \in \Sigma_{k}(x)$. Since $\varphi \in \mathrm{U}_{k+2}$, by our assumption, there exists a sentence $\varphi^{\prime} \in \Pi_{k+1}$ such that $T \vdash \varphi \leftrightarrow \varphi^{\prime}$. Since PA $\vdash \varphi$, we have PA $\vdash \varphi^{\prime}$. Then, by Theorem 6.14, we have HA $+\Sigma_{k-1}$-LEM $\vdash \varphi^{\prime}$, and hence, $T \vdash \varphi^{\prime}$. Thus we have $T \vdash \varphi$.

Theorem 7.3. Let $T$ be a theory in-between $\mathrm{HA}$ and PA. Then $T \vdash\left(\Pi_{k} \vee \Pi_{k}\right)$-DNE if and only if $\operatorname{PNFT}_{T}\left(\mathrm{U}_{k^{\prime}}, \Pi_{k^{\prime}}\right)$ for all $k^{\prime} \leq k$.

Proof. The "only if" direction is immediate from Theorem 5.3,(2) and Lemma 2.3. We show the converse direction. Assume $\operatorname{PNFT}_{T}\left(\mathrm{U}_{k^{\prime}}, \Pi_{k^{\prime}}\right)$ for all $k^{\prime} \leq k$. Let $k>0$ without loss of generality. By Lemma 7.2, $T \vdash \Sigma_{k-1}$-LEM. Then, by Lemma 7.1, we have $T \vdash\left(\Pi_{k} \vee \Pi_{k}\right)$-DNE.

Definition 7.4. Let $\Gamma$ be a class of formulas. Then $\Gamma^{\text {dn }}$ denotes the class of HA-formulas $\neg \neg \varphi$ where $\varphi \in \Gamma$, and $\Gamma^{\mathrm{n}}$ denotes that for $\neg \varphi$ where $\varphi \in \Gamma$.

Lemma 7.5. Let $T$ be a theory in-between $\mathrm{HA}$ and $\mathrm{PA}$. If $\mathrm{PNFT}_{T}\left(\mathrm{U}_{k^{\prime}}{ }^{\mathrm{dn}}, \Pi_{k^{\prime}} \mathrm{dn}\right)$ for all $k^{\prime} \leq k$, then $T \vdash \neg \neg \Sigma_{k-1}$-LEM.

Proof. By induction on $k$. The base case is trivial. For the induction step, assume $\operatorname{PNFT}_{T}\left(\mathrm{U}_{k^{\prime}}{ }^{\mathrm{dn}}, \Pi_{k^{\prime}}{ }^{\mathrm{dn}}\right)$ for all $k^{\prime} \leq k+1$. Then, by induction hypothesis, $T$ proves $\neg \neg \Sigma_{k-1}$-LEM. Fix an instance of $\neg \neg \Sigma_{k}$-LEM

$$
\varphi: \equiv \neg \neg \forall x\left(\varphi_{1}(x) \vee \neg \varphi_{1}(x)\right)
$$

where $\varphi_{1}(x) \in \Sigma_{k}(x)$. Since $\varphi \in \mathrm{U}_{k+1}$ dn, by our assumption, there exists a sentence $\varphi^{\prime} \in \Pi_{k+1}$ such that $T \vdash \varphi \leftrightarrow \neg \neg \varphi^{\prime}$. Since PA $\vdash \forall x\left(\varphi_{1}(x) \vee\right.$ $\left.\neg \varphi_{1}(x)\right)$, we have PA $\vdash \varphi^{\prime}$. Then, by Theorem 6.14, we have HA $+\Sigma_{k-1}$-LEM $\vdash$ $\varphi^{\prime}$, and hence, HA $+\neg \neg \Sigma_{k-1}$-LEM $\vdash \neg \neg \varphi^{\prime}$ by Lemma 4.1, Then $T \vdash \varphi$.

Theorem 7.6. Let $T$ be a theory in-between $\mathrm{HA}$ and PA. The following are pairwise equivalent:

1. $\operatorname{PNFT}_{T}\left(\mathrm{E}_{k^{\prime}}{ }^{\mathrm{n}}, \Pi_{k^{\prime}}{ }^{\mathrm{dn}}\right)$ for all $k^{\prime} \leq k$;

2. $\operatorname{PNFT}_{T}\left(\mathrm{U}_{k^{\prime}}{ }^{\mathrm{dn}}, \Pi_{k^{\prime}} \mathrm{dn}\right)$ for all $k^{\prime} \leq k$;

3. $T \vdash \mathrm{U}_{k}^{+}-\mathrm{DNS}$.

Proof. The equivalence of (11) and (2) is trivial (cf. the proof of Lemma 5.1). In addition, $(3 \rightarrow 2)$ is immediate from Lemma 5.1. In what follows, we show (2 $\rightarrow 3)$. Assume $\operatorname{PNFT}_{T}\left(\mathrm{U}_{k^{\prime}} \mathrm{dn}, \Pi_{k^{\prime}} \mathrm{dn}\right)$ for all $k^{\prime} \leq k$. Since $\mathrm{U}_{k}^{+}$-DNS is intuitionistically equivalent to $\neg \neg \mathrm{U}_{k}^{+}$-DNS (See Remark 2.8), it suffices to show $T \vdash \neg \neg \mathrm{U}_{k}^{+}$-DNS. Let $k>0$ without loss of generality. Fix an instance of $\neg \neg \mathrm{U}_{k}^{+}$-DNS

$$
\varphi: \equiv \neg \neg \forall x\left(\forall y \neg \neg \varphi_{1}(x, y) \rightarrow \neg \neg \forall y \varphi_{1}(x, y)\right)
$$


where $\varphi_{1}(x, y) \in \mathrm{U}_{k}^{+}(x, y)$. By Lemma 4.5, we have that $\forall y \neg \neg \varphi_{1}(x, y)$ and $\forall y \varphi_{1}(x, y)$ are in $\mathrm{U}_{k}^{+}(x)$. Since $i(s) \equiv-$ for all alternation paths $s$ of $\forall y \neg \neg \varphi_{1}(x, y)$ and $\forall y \varphi_{1}(x, y)$, it is straightforward to show that there exists $k^{\prime} \leq k$ such that $\forall y \neg \neg \varphi_{1}(x, y)$ and $\forall y \varphi_{1}(x, y)$ are in $\mathrm{U}_{k^{\prime}}(x)$. Then, by $\operatorname{PNFT}_{T}\left(\mathrm{U}_{k^{\prime}} \mathrm{dn}, \Pi_{k^{\prime}} \mathrm{dn}\right)$, there exist $\rho(x), \rho^{\prime}(x) \in \Pi_{k^{\prime}}(x)$ such that $T$ proves $\neg \neg \rho(x) \leftrightarrow \neg \neg \forall y \varphi_{1}(x, y)$ and $\neg \neg \rho^{\prime}(x) \leftrightarrow \neg \neg \forall y \neg \neg \varphi_{1}(x, y)$. Since PA is an extension of $T$ and PA $\vdash \varphi$, we have PA $\vdash \rho^{\prime}(x) \rightarrow \rho(x)$. Then, by Theorem 6.14. we have that HA $+\Sigma_{k^{\prime}-2}$-LEM (HA if $\left.k^{\prime}<2\right)$ proves $\rho^{\prime}(x) \rightarrow \rho(x)$, and hence, $\forall x\left(\neg \neg \rho^{\prime}(x) \rightarrow \neg \neg \rho(x)\right)$. By Lemma 4.1, we have

$$
\mathrm{HA}+\neg \neg \Sigma_{k^{\prime}-2} \text { LEM } \vdash \neg \neg \forall x\left(\neg \neg \rho^{\prime}(x) \rightarrow \neg \neg \rho(x)\right) .
$$

On the other hand, by Lemma 7.5 and our assumption, we have $T \vdash$ $\neg \neg \Sigma_{k-1}$-LEM. Then we have

$$
T \vdash \neg \neg \forall x\left(\neg \neg \forall y \neg \neg \varphi_{1}(x, y) \rightarrow \neg \neg \forall y \varphi_{1}(x, y)\right),
$$

and hence, $T \vdash \varphi$.

Remark 7.7. Theorem 7.6 shows that the verification theory for Lemma 5.1 is optimal.

Definition 7.8. Let $\Gamma$ be a class of HA-formulas. $\Gamma^{\mathrm{df}}$ denotes the class of formulas in $\Gamma$ which do not contain $\vee$.

Lemma 7.9. Let $T$ be a theory in-between $\mathrm{HA}+\Pi_{k-1}$-LEM ( $\mathrm{HA}$ if $k=0$ ) and PA. If $\mathrm{PNFT}_{T}\left(\mathrm{E}_{k^{\prime}} \mathrm{df}, \Sigma_{k^{\prime}}\right)$ for all $k^{\prime} \leq k$, then $T \vdash \Sigma_{k}$-DNE.

Proof. By induction on $k$. The base case is trivial. For the induction step, assume the assertion for $k$ and let $T$ be a theory in-between $\mathrm{HA}+\Pi_{k}$-LEM and PA. Assume also that $\mathrm{PNFT}_{T}\left(\mathrm{E}_{k^{\prime}} \mathrm{df}, \Sigma_{k^{\prime}}\right)$ holds for all $k^{\prime} \leq k+1$. Then, by induction hypothesis, $T$ proves $\Sigma_{k}$-DNE. Since $T$ contains $\mathrm{HA}+\Pi_{k}$-LEM, we have $T \vdash \Sigma_{k}$-LEM by [1, Theorem 3.1(ii)]. Fix an instance of $\Sigma_{k+1}$-DNE

$$
\varphi: \equiv \forall x\left(\neg \neg \varphi_{1}(x) \rightarrow \varphi_{1}(x)\right),
$$

where $\varphi_{1}(x) \in \Sigma_{k+1}(x)$. Without loss of generality, one can assume that $\varphi_{1}(x)$ does not contain $\vee\left(\right.$ cf. [5, Proposition 3.8]). Since $\neg \neg \varphi_{1}(x) \in \mathrm{E}_{k+1} \mathrm{df}$, By $\operatorname{PNFT}_{T}\left(\mathrm{E}_{k+1} \mathrm{df}, \Sigma_{k+1}\right)$, there exists $\varphi_{1}^{\prime}(x) \in \Sigma_{k+1}(x)$ such that $T \vdash$ $\neg \neg \varphi_{1}(x) \leftrightarrow \varphi_{1}^{\prime}(x)$. Since PA is an extension of $T$ and PA $\vdash \varphi$, we have PA $\vdash \varphi_{1}^{\prime}(x) \rightarrow \varphi_{1}(x)$. Then, by Lemma 2.3 and Theorem 6.14, we have that $\mathrm{HA}+\Sigma_{k}$-LEM proves $\varphi_{1}^{\prime}(x) \rightarrow \varphi_{1}(x)$, and hence, $\forall x\left(\varphi_{1}^{\prime}(x) \rightarrow \varphi_{1}(x)\right)$. Since $T$ is an extension of $\mathrm{HA}+\Sigma_{k}$-LEM, we have $T \vdash \varphi$.

Lemma 7.10. Let $T$ be an extension of $\mathrm{HA}+\neg \neg \Sigma_{k-1}$-DNE (HA if $\left.k=0\right)$. If $\operatorname{PNFT}_{T}\left(\mathrm{E}_{k^{\prime}}, \Sigma_{k^{\prime}}\right)$ for all $k^{\prime} \leq k$, then $\operatorname{PNFT}_{T}\left(\mathrm{E}_{k^{\prime}}{ }^{\mathrm{n}}, \Pi_{k^{\prime}} \mathrm{dn}\right)$ for all $k^{\prime} \leq k$. 
Proof. Assume $\mathrm{PNFT}_{T}\left(\mathrm{E}_{k^{\prime}}, \Sigma_{k^{\prime}}\right)$ for all $k^{\prime} \leq k$. Fix $k^{\prime} \leq k$ and $\varphi \in \mathrm{E}_{k^{\prime}}$. By $\operatorname{PNFT}_{T}\left(\mathrm{E}_{k^{\prime}}, \Sigma_{k^{\prime}}\right)$, there exists $\varphi^{\prime} \in \Sigma_{k^{\prime}}$ such that $\mathrm{FV}(\varphi)=\mathrm{FV}\left(\varphi^{\prime}\right)$ and $T \vdash \varphi \leftrightarrow \varphi^{\prime}$. Then

$$
T \vdash \neg \varphi \leftrightarrow \neg \varphi^{\prime} .
$$

On the other hand, by Lemma 4.7.(2), there exists $\varphi^{\prime \prime} \in \Pi_{k^{\prime}}$ such that $\mathrm{FV}\left(\varphi^{\prime}\right)=\mathrm{FV}\left(\varphi^{\prime \prime}\right)$ and $\mathrm{HA}+\Sigma_{k^{\prime}-1}$-DNE $\vdash \neg \varphi^{\prime} \leftrightarrow \varphi^{\prime \prime}$. Then, by Corollary 4.2, we have

$$
\mathrm{HA}+\neg \neg \Sigma_{k^{\prime}-1}-\mathrm{DNE} \vdash \neg \varphi^{\prime} \leftrightarrow \neg \neg \varphi^{\prime \prime} .
$$

Then FV $(\neg \varphi)=\mathrm{FV}\left(\neg \neg \varphi^{\prime \prime}\right)$ and $T \vdash \neg \varphi \leftrightarrow \neg \neg \varphi^{\prime \prime}$. Thus we have shown $\operatorname{PNFT}_{T}\left(\mathrm{E}_{k^{\prime}}{ }^{\mathrm{n}}, \Sigma_{k^{\prime}} \mathrm{dn}\right)$.

Theorem 7.11. Let $T$ be a theory in-between $\mathrm{HA}+\Pi_{k-1}$-LEM ( $\mathrm{HA}$ if $\left.k=0\right)$ and $\mathrm{PA}$. Then $T \vdash \Sigma_{k}$-DNE $+\mathrm{U}_{k}^{+}$-DNS if and only if $\mathrm{PNFT}_{T}\left(\mathrm{E}_{k^{\prime}}, \Sigma_{k^{\prime}}\right)$ for all $k^{\prime} \leq k$.

Proof. The "only if" direction is immediate from Theorem 5.3. (1) and Lemma 2.3. We show the converse direction. Assume $\operatorname{PNFT}_{T}\left(\mathrm{E}_{k^{\prime}}, \Sigma_{k^{\prime}}\right)$ for all $k^{\prime} \leq k$. Let $k>0$ without loss of generality. By Lemma [7.9, we have $T \vdash \Sigma_{k}$-DNE. In addition, by Lemma 7.10 and Theorem 7.6, we have $T \vdash \mathrm{U}_{k}^{+}$-DNS.

Remark 7.12. It is still open whether the assumption that $T$ contains $\Pi_{k-1}$-LEM can be omitted in Theorem 7.11,

Remark 7.13. Akama et al. [1] shows that $\Pi_{k}$-LEM does not derive $\Sigma_{k}$-DNE and $\Sigma_{k}$-DNE does not derive $\left(\Pi_{k} \vee \Pi_{k}\right)$-DNE. Theorem 7.11 reveals that the prenex normal form theorem for $\mathrm{E}_{k}$ and $\Sigma_{k}$ does not hold in $\mathrm{HA}+\Pi_{k}-\mathrm{LEM}$, and Theorem 7.3 reveals that the prenex normal form theorem for $\mathrm{U}_{k}$ and $\Pi_{k}$ does not hold in $\mathrm{HA}+\Sigma_{k}$-DNE.

Corollary 7.14. Let $T$ be a theory in-between $\mathrm{HA}$ and PA. Then $T \vdash$ $\Sigma_{k}-\mathrm{DNE}+\left(\Pi_{k} \vee \Pi_{k}\right)-\mathrm{DNE}$ if and only if $\mathrm{PNFT}_{T}\left(\mathrm{E}_{k^{\prime}}, \Sigma_{k^{\prime}}\right)$ and $\mathrm{PNFT}_{T}\left(\mathrm{U}_{k^{\prime}}, \Pi_{k^{\prime}}\right)$ for all $k^{\prime} \leq k$.

Proof. Let $T$ be a theory in-between HA and PA. The "only if" direction follows from Theorem 5.3, Corollary 5.4 and Lemma 2.3,

For the converse direction, assume that $\mathrm{PNFT}_{T}\left(\mathrm{E}_{k^{\prime}}, \Sigma_{k^{\prime}}\right)$ and $\mathrm{PNFT}_{T}\left(\mathrm{U}_{k^{\prime}}, \Pi_{k^{\prime}}\right)$ hold for all $k^{\prime} \leq k$. By Theorems 7.3, we have $T \vdash\left(\Pi_{k} \vee \Pi_{k}\right)$-DNE. Since $\Pi_{k-1}$-LEM is derived from $\left(\Pi_{k} \vee \Pi_{k}\right)$-DNE (cf. [1, Theorem 3.1(1)]), by Theorem 7.11, we also have $T \vdash \Sigma_{k}$-DNE.

In the following, we show the optimality of Theorem 5.7 (see Theorem 7.16).

Lemma 7.15. Let $T$ be a theory in-between $\mathrm{HA}+\Pi_{k-2}$-LEM ( $\mathrm{HA}$ if $k<2$ ) and $\mathrm{PA}$. If $\mathrm{PNFT}_{T}\left(\left(\mathrm{U}_{k^{\prime}} \mathrm{dn}\right)^{\mathrm{df}}, \Pi_{k^{\prime}}\right)$ for all $k^{\prime} \leq k$, then $T \vdash \Sigma_{k-1}$-DNE. 
Proof. By induction on $k$. The base case is trivial. For the induction step, assume the assertion for $k$ and let $T$ be a theory in-between $\mathrm{HA}+\Pi_{k-1}$-LEM and PA. Assume also that $\operatorname{PNFT}_{T}\left(\left(\mathrm{U}_{k^{\prime}} \mathrm{dn}\right)^{\mathrm{df}}, \Pi_{k^{\prime}}\right)$ holds for all $k^{\prime} \leq k+$ 1. Then, by induction hypothesis, $T$ proves $\Sigma_{k-1}$-DNE. Since $T$ contains HA $+\Pi_{k-1}$-LEM, we have $T \vdash \Sigma_{k-1}$-LEM by [1, Theorem 3.1(ii)]. Fix an instance of $\Sigma_{k}$-DNE

$$
\varphi: \equiv \forall x\left(\neg \neg \varphi_{1}(x) \rightarrow \varphi_{1}(x)\right),
$$

where $\varphi_{1}(x) \in \Sigma_{k}(x)$. Without loss of generality, one can assume that $\varphi_{1}(x)$ does not contain $\vee$ (cf. [5, Proposition 3.8]). From the perspective of Remark 2.5. $\varphi_{1}(x)$ is in $\Pi_{k+1}(x)$. Then, by $\operatorname{PNFT}_{T}\left(\left(\mathrm{U}_{k+1} \mathrm{dn}\right)^{\mathrm{df}}, \Pi_{k+1}\right)$, there exists $\varphi_{1}^{\prime}(x) \in \Pi_{k}(x)$ such that $T \vdash \neg \neg \varphi_{1}(x) \leftrightarrow \varphi_{1}^{\prime}(x)$. Since PA is an extension of $T$ and PA $\vdash \varphi$, we have PA $\vdash \varphi_{1}^{\prime}(x) \rightarrow \varphi_{1}(x)$. By Theorem 6.14, we have that HA $+\Sigma_{k-1}$-LEM proves $\varphi_{1}^{\prime}(x) \rightarrow \varphi_{1}(x)$, and hence, $\forall x\left(\varphi_{1}^{\prime}(x) \rightarrow \varphi_{1}(x)\right)$. Since $T$ is an extension of HA $+\Sigma_{k-1}$-LEM, we have $T \vdash \varphi$.

\section{Theorem 7.16.}

1. Let $T$ be a theory in-between $\mathrm{HA}+\Pi_{k-1}-\mathrm{LEM}(\mathrm{HA}$ if $k=0)$ and PA. Then $T \vdash \Sigma_{k}$-DNE if and only if $\operatorname{PNFT}_{T}\left(\mathrm{E}_{k^{\prime}} \mathrm{df}, \Sigma_{k^{\prime}}\right)$ for all $k^{\prime} \leq k$.

2. Let $T$ be a theory in-between $\mathrm{HA}+\Pi_{k-2}$-LEM ( $\mathrm{HA}$ if $k<2$ ) and PA. Then $T \vdash \Sigma_{k-1}-\mathrm{DNE}$ if and only if $\operatorname{PNFT}_{T}\left(\mathrm{U}_{k^{\prime}} \mathrm{df}, \Pi_{k^{\prime}}\right)$ for all $k^{\prime} \leq k$.

Proof. (11): The "only if" direction is by Theorem 5.7,(11). The converse direction is by Lemma 7.9.

(2): The "only if" direction is by Theorem [5.7.(2). Note that any formula in $\left(\mathrm{U}_{k^{\prime}} \mathrm{dn}\right) \mathrm{df}$ is in $\mathrm{U}_{k^{\prime}}{ }^{\mathrm{df}}$. Then the converse direction follows from Lemma 7.15 .

At the end of this section, we characterize some variants of prenex normal form theorems.

Theorem 7.17. Let $T$ be a theory in-between $\mathrm{HA}+\Pi_{k-2}$-LEM $(\mathrm{HA}$ if $k<2)$ and PA. Then $T \vdash \Sigma_{k-1}$-DNE $+\mathrm{U}_{k}^{+}$-DNS if and only if $\mathrm{PNFT}_{T}\left(\mathrm{U}_{k^{\prime}} \mathrm{dn}^{\mathrm{n}}, \Pi_{k^{\prime}}\right)$ for all $k^{\prime} \leq k$.

Proof. We first show the "only if" direction. Let $k>0$ without loss of generality. Assume $T \vdash \Sigma_{k-1}$-DNE $+\mathrm{U}_{k}^{+}$-DNS and fix $k^{\prime} \leq k$. Since $T \vdash$ $\mathrm{U}_{k^{\prime}}^{+}$DNS, by Lemma 5.1, for any $\varphi \in \mathrm{U}_{k^{\prime}}^{+}$, there exists $\varphi^{\prime} \in \Pi_{k^{\prime}}$ such that $\mathrm{FV}(\varphi)=\mathrm{FV}\left(\varphi^{\prime}\right)$ and

$$
T \vdash \neg \neg \varphi \leftrightarrow \neg \neg \varphi^{\prime} .
$$

Since $T \vdash \Sigma_{k-1}$-DNE, by Lemma [5.2, (44), we have $T \vdash \neg \neg \varphi^{\prime} \leftrightarrow \varphi^{\prime}$, and hence, $T \vdash \neg \neg \varphi \leftrightarrow \varphi^{\prime}$. Thus we have $\operatorname{PNFT}_{T}\left(\mathrm{U}_{k^{\prime}} \mathrm{dn}, \Pi_{k^{\prime}}\right)$. 
Next, we show the converse direction. Assume that $\operatorname{PNFT}_{T}\left(\mathrm{U}_{k^{\prime}}{ }^{\mathrm{dn}}, \Pi_{k^{\prime}}\right)$ holds for all $k^{\prime} \leq k$. By Lemma 7.15, we have $T \vdash \Sigma_{k-1}$-DNE. On the other hand, by the assumption, we have $\operatorname{PNFT}_{T}\left(\mathrm{U}_{k^{\prime}}{ }^{\mathrm{dn}}, \Pi_{k^{\prime}} \mathrm{dn}\right)$ for all $k^{\prime} \leq k$, and hence, $T \vdash \mathrm{U}_{k}^{+}$-DNS by Theorem 7.6 .

Theorem 7.18. Let $T$ be a theory in-between $\mathrm{HA}+\neg \neg \Pi_{k-2}$-LEM (HA if $k<2$ ) and PA. The following are pairwise equivalent:

1. $\operatorname{PNFT}_{T}\left(\left(\mathrm{E}_{k^{\prime}}{ }^{\mathrm{n}}\right)^{\mathrm{df}}, \Pi_{k^{\prime}} \mathrm{dn}\right)$ for all $k^{\prime} \leq k$;

2. $\operatorname{PNFT}_{T}\left(\left(\mathrm{U}_{k^{\prime}}{ }^{\mathrm{dn}}\right)^{\mathrm{df}}, \Pi_{k^{\prime}} \mathrm{dn}\right)$ for all $k^{\prime} \leq k$;

3. $T \vdash \neg \neg \Sigma_{k-1}$-DNE.

Proof. The equivalence of (1) and (2) is trivial (cf. the proof of Lemma 5.1). In addition, $(3-1)$ is immediate from the item 3 in the proof of Theorem 5.7. Then it suffices to show $(1-3)$. We show this by induction on $k$. The base case is trivial. For the induction step, assume the assertion for $k$ and let $T$ be a theory in-between HA $+\neg \neg \Pi_{k-1}$-LEM and PA. Assume also that $\left.\operatorname{PNFT}_{T}\left(\left(\mathrm{E}_{k^{\prime}}\right)^{\mathrm{n}}\right)^{\mathrm{df}}, \Pi_{k^{\prime}} \mathrm{dn}\right)$ holds for all $k^{\prime} \leq k+1$. Then, by induction hypothesis, we have $T \vdash \neg \neg \Sigma_{k-1}$-DNE. Since $T$ contains HA $+\neg \neg \Pi_{k-1}$-LEM, we have $T \vdash \neg \neg \Sigma_{k-1}$-LEM by [1, Theorem 3.1(ii)]. Fix an instance of $\neg \neg \Sigma_{k}$-DNE

$$
\varphi: \equiv \neg \neg \forall x\left(\neg \neg \varphi_{1}(x) \rightarrow \varphi_{1}(x)\right),
$$

where $\varphi_{1}(x) \in \Sigma_{k}(x)$. Without loss of generality, one can assume that $\varphi_{1}(x)$ does not contain $\vee$ (cf. [5, Proposition 3.8]). Note $\forall x\left(\neg \neg \varphi_{1}(x) \rightarrow \varphi_{1}(x)\right) \in$ $\mathrm{U}_{k+1} \mathrm{df}$, and hence, $\left.\varphi \in\left(\mathrm{E}_{k+1}\right)^{\mathrm{n}}\right)^{\mathrm{df}}$. By $\operatorname{PNFT}_{T}\left(\left(\mathrm{E}_{k+1}\right)^{\mathrm{n}}{ }^{\mathrm{df}}, \Pi_{k^{\prime}} \mathrm{dn}\right)$, there exists a sentence $\varphi^{\prime} \in \Pi_{k+1}$ such that $T \vdash \varphi \leftrightarrow \neg \neg \varphi^{\prime}$. Since PA is an extension of $T$ and PA $\vdash \varphi$, we have PA $\vdash \varphi^{\prime}$. By Theorem 6.14, we have $\mathrm{HA}+\Sigma_{k-1}-\mathrm{LEM} \vdash \varphi^{\prime}$. Then, by Lemma 4.1, we have

$$
\mathrm{HA}+\neg \neg \Sigma_{k-1}-\mathrm{LEM} \vdash \neg \neg \varphi^{\prime} .
$$

Since $T$ is an extension of HA $+\neg \neg \Sigma_{k-1}$-LEM, we have $T \vdash \varphi$.

All of our characterization results are of the following form: For any theory $T$ in-between $\mathrm{HA}+\mathrm{Q}_{k}$ and PA, $T \vdash \mathrm{P}_{k}$ if and only if $\mathrm{PNFT}_{T}\left(\Gamma_{k^{\prime}}, \Delta_{k^{\prime}}\right)$ holds for all $k^{\prime} \leq k$, where $\mathrm{P}_{k}, \mathrm{Q}_{k}$ are logical principles and $\Gamma_{k^{\prime}}, \Delta_{k^{\prime}}$ are classes of formulas. Based on this representation, our results are summarized in Table 1, where it is also possible to replace $\mathrm{U}_{k}^{+}$-DNS by $\mathrm{U}_{k}$-DNS (see Remark 4.11). 


\begin{tabular}{|c|c|c|c|}
\hline $\mathrm{P}_{k}$ & $\left(\Gamma_{k}, \Delta_{k}\right)$ & $\mathrm{Q}_{k}$ & \\
\hline$\neg \neg \Sigma_{k-1}-\mathrm{DNE}$ & $\overline{\left(\left(\left(\mathrm{U}_{k} \mathrm{dn}\right)^{\mathrm{df}}, \Pi_{k} \mathrm{dn}\right)\right.}$ & $\neg \neg \Pi_{k-2^{-}} \mathrm{LEM}$ & Theorem 7.18 \\
\hline $\mathrm{U}_{k}^{+}-\mathrm{DNS}$ & $\left(\mathrm{U}_{k}^{\mathrm{dn}}, \Pi_{k}^{\mathrm{dn}}\right)$ & $\emptyset$ & Theorem 7.6 \\
\hline$\Sigma_{k-1}$-DNE & $\left(\mathrm{U}_{k}^{\mathrm{df}}, \Pi_{k}\right)$ & $\Pi_{k-2}$-LEM & Theorem 7.16. (2) \\
\hline$\Sigma_{k-1}-\mathrm{DNE}+\mathrm{U}_{k}^{+}-\mathrm{DNS}$ & $\left(\mathrm{U}_{k}^{\mathrm{dn}}, \Pi_{k}\right)$ & $\Pi_{k-2}$-LEM & Theorem 7.17 \\
\hline$\Sigma_{k}-\mathrm{DNE}$ & $\left(\mathrm{E}_{k}^{\mathrm{df}}, \Sigma_{k}\right)$ & $\Pi_{k-1}$-LEM & Theorem 7.16. (1) \\
\hline$\Sigma_{k}-\mathrm{DNE}+\mathrm{U}_{k}^{+}-\mathrm{DNS}$ & $\left(\mathrm{E}_{k}, \Sigma_{k}\right)$ & $\Pi_{k-1}$-LEM & Theorem 7.11 \\
\hline$\left(\Pi_{k} \vee \Pi_{k}\right)-\mathrm{DNE}$ & $\left(\mathrm{U}_{k}, \Pi_{k}\right)$ & $\emptyset$ & Theorem 7.3 \\
\hline$\Sigma_{k}-\mathrm{DNE}+\left(\Pi_{k} \vee \Pi_{k}\right)-\mathrm{DNE}$ & $\left(\mathrm{U}_{k}, \Pi_{k}\right) \&\left(\mathrm{E}_{k}, \Sigma_{k}\right)$ & $\emptyset$ & Corollary 7.14 \\
\hline
\end{tabular}

Table 1: Characterizations of the prenex normal form theorems

\section{Acknowledgements}

The authors thank to Ulrich Kohlenbach for his helpful comments. The first author was supported by JSPS KAKENHI Grant Numbers JP18K13450 and JP19J01239, and the second author by JP19K14586.

\section{References}

[1] Y. Akama, S. Berardi, S. Hayashi, and U. Kohlenbach. An arithmetical hierarchy of the law of excluded middle and related principles. In Proceedings of the 19th Annual IEEE Symposium on Logic in Computer Science (LICS'04), pages 192-301. 2004.

[2] H. Friedman. Classically and intuitionistically provably recursive functions. In G. H. Müller and D. S. Scott, editors, Higher Set Theory, pages 21-27, Berlin, Heidelberg, 1978. Springer Berlin Heidelberg.

[3] M. Fujiwara and U. Kohlenbach. Interrelation between weak fragments of double negation shift and related principles. J. Symb. Log., 83(3):991$1012,2018$.

[4] M. Fujiwara and T. Kurahashi. Conservation results on semi-classical arithmetic. preprint.

[5] U. Kohlenbach. Applied proof theory: proof interpretations and their use in mathematics. Springer Monographs in Mathematics. Springer-Verlag, Berlin, 2008. 
[6] U. Kohlenbach and P. Safarik. Fluctuations, effective learnability and metastability in analysis. Annals of Pure and Applied Logic, 165(1):266 - 304, 2014. The Constructive in Logic and Applications.

[7] A. S. Troelstra, editor. Metamathematical investigation of intuitionistic arithmetic and analysis, volume 344 of Lecture Notes in Mathematics. Springer-Verlag, Berlin, New York, 1973.

[8] A. S. Troelstra and D. van Dalen. Constructivism in mathematics, An introduction, Vol. I, volume 121 of Studies in Logic and the Foundations of Mathematics. North Holland, Amsterdam, 1988.

[9] D. van Dalen. Logic and Structure. Universitext. Springer-Verlag London, fifth edition, 2013. 